\title{
Using flow cytometry and light-induced fluorescence to characterize the variability and characteristics of bioaerosols in springtime in Metro Atlanta, Georgia
}

\author{
Arnaldo Negron ${ }^{1,2}$, Natasha DeLeon-Rodriguez ${ }^{3, a}$, Samantha M. Waters ${ }^{1, b}$, Luke D. Ziemba ${ }^{4}$, Bruce Anderson $^{4}$, \\ Michael Bergin ${ }^{5}$, Konstantinos T. Konstantinidis ${ }^{6,3}$, and Athanasios Nenes ${ }^{1,7,8}$ \\ ${ }^{1}$ School of Earth and Atmospheric Sciences, Georgia Institute of Technology, Atlanta, GA 30332, USA \\ ${ }^{2}$ School of Chemical and Biomolecular Engineering, Georgia Institute of Technology, Atlanta, GA 30332, USA \\ ${ }^{3}$ School of Biology, Georgia Institute of Technology, Atlanta, GA 30332, USA \\ ${ }^{4}$ School of Biological Sciences, Chemistry and Dynamics Branch/Science Directorate, National Aeronautics and Space \\ Administration Langley Research Center, Hampton, VA 23681, USA \\ ${ }^{5}$ Department of Civil and Environmental Engineering, Duke University, Durham, NC 2770, USA \\ ${ }^{6}$ School of Civil and Environmental Engineering, Georgia Institute of Technology, Atlanta, GA 30332, USA \\ ${ }^{7}$ Institute for Chemical Engineering Science, Foundation for Research and Technology Hellas, Patra, 26504, Greece \\ ${ }^{8}$ Laboratory of Atmospheric Processes and their Impacts (LAPI), School of Architecture, Civil \& Environmental Engineering, \\ Ecole Polytechnique Fédérale de Lausanne, Lausanne, 1015, Switzerland \\ ${ }^{a}$ currently at: Puerto Rico Science, Technology and Research Trust, Rio Piedras, 00927, Puerto Rico \\ b currently at: Department of Marine Sciences, University of Georgia, Athens, GA 30602-3636, USA
}

Correspondence: Konstantinos T. Konstantinidis (kostas.konstantinidis@gatech.edu) and Athanasios Nenes (athanasios.nenes@epfl.ch)

Received: 9 October 2018 - Discussion started: 30 October 2018

Revised: 12 September 2019 - Accepted: 22 September 2019 - Published: 14 February 2020

\begin{abstract}
The abundance and speciation of primary biological aerosol particles (PBAP) is important for understanding their impacts on human health, cloud formation, and ecosystems. Towards this, we have developed a protocol for quantifying PBAP collected from large volumes of air with a portable wet-walled cyclone bioaerosol sampler. A flow cytometry (FCM) protocol was then developed to quantify and characterize the PBAP populations from the sampler, which were confirmed against epifluorescence microscopy. The sampling system and FCM analysis were used to study PBAP in Atlanta, GA, over a 2-month period and showed clearly defined populations of nucleic-acid-containing particles: low nucleic acid-content particles above threshold (LNA-AT) and high nucleic acid-content particles (HNA) likely containing wet-ejected fungal spores and pollen. We find that the dailyaverage springtime PBAP concentration ( 1 to $5 \mu \mathrm{m}$ diameter) ranged between $1.4 \times 10^{4}$ and $1.1 \times 10^{5} \mathrm{~m}^{-3}$. The LNA-AT population dominated PBAP during dry days $(72 \pm 18 \%)$;
\end{abstract}

HNA dominated the PBAP during humid days and following rain events, where HNA comprised up to $92 \%$ of the PBAP number. Concurrent measurements with a Wideband Integrated Bioaerosol Sensor (WIBS-4A) showed that fluorescent biological aerosol particles (FBAP) and total FCM counts are similar; HNA (from FCM) moderately correlated with ABC-type FBAP concentrations throughout the sampling period (and for the same particle size range, $1-5 \mu \mathrm{m}$ diameter). However, the FCM LNA-AT population, possibly containing bacterial cells, did not correlate with any FBAP type. The lack of correlation of any WIBS FBAP type with the LNA-AT suggests that airborne bacterial cells may be more difficult to unambiguously detect with autofluorescence than currently thought. Identification of bacterial cells even in the FCM (LNA-AT population) is challenging, given that the fluorescence level of stained cells at times may be comparable to that seen from abiotic particles. HNA and ABC displayed the highest concentration on a humid and warm 
day after a rain event (14 April 2015), suggesting that both populations correspond to wet-ejected fungal spores. Overall, information from both instruments combined reveals a highly dynamic airborne bioaerosol community over Atlanta, with a considerable presence of fungal spores during humid days and an LNA-AT population dominating the bioaerosol community during dry days.

\section{Introduction}

Primary biological aerosol particles (PBAP), also called bioaerosols, are comprised of airborne microbial cells (e.g., bacteria, diatoms), reproductive entities (e.g., pollen, fungal spores), viruses, and biological fragments. Bioaerosols are ubiquitous, with potentially important impacts on human health, cloud formation, precipitation, and biogeochemical cycles (Pöschl, 2005; Hoose et al., 2010; DeLeon-Rodriguez et al., 2013; Morris et al., 2014; Longo et al., 2014; FröhlichNowoisky et al., 2016; Myriokefalitakis et al., 2016). Despite their low number concentration relative to abiotic particles, PBAP possess unique functional and compositional characteristics that differentiate them from abiotic aerosol. For example, certain PBAP constitute the most efficient of atmospheric ice nucleators, affecting the microphysics of mixed phase clouds and precipitation (Hoose and Möhler, 2012; Sullivan et al., 2018). The mass and nutrient content of PBAP may suffice to comprise an important supply of bioavailable phosphorous to oligotrophic marine ecosystems (Longo et al., 2014; Myriokefalitakis et al., 2016). In addition, the concurrence of disease outbreaks during dust storms has been attributed to pathogenic microbes attached to airborne dust that are subsequently inhaled (Griffin et al., 2003; Ortiz-Martínez et al., 2015; Goudie, 2014).

Quantification of the concentration and size of PBAP is critical for understanding their environmental impacts. Measuring PBAP however poses a challenge for established microbiology tools, owing to their low atmospheric concentration $\left(10^{3}-10^{6}\right.$ cells $^{-3}$ air; Fröhlich-Nowoisky et al., 2016) and wide diversity of airborne particle types and sizes. For instance, only a fraction of microorganisms (an estimated $5 \%$; Chi and $\mathrm{Li}, 2007$ ) can be cultured, and cultivation cannot be used to quantify dead organisms, viruses, or fragments, while most culture-independent methods are optimized for more abundant microbial populations. Epifluorescence microscopy (EPM) is the standard for bioaerosol quantification, but is not high throughput and requires considerable time for quantification of a concentration per sample. Flow cytometry (FCM) is an analysis technique based on the concurrent measurement of light scattering and fluorescence intensity from single particles (Wang et al., 2010). FCM requires a liquid suspension of bioparticles that flows through an optical cell and is interrogated with a series of laser beams. Each sample is pretreated with stains that target specific macro- molecules (e.g., DNA/RNA) which subsequently fluoresce when excited by the FCM lasers. The resulting scattering and fluorescent light emissions are then detected by an array of sensors to allow the differentiation of biological and abiotic (e.g., dust) particles according to the characteristic specific to the stain used. FCM has proven to be as reliable as EPM, but with the advantage of lower uncertainty, higher quantification efficiency, and requiring considerably less time and effort than EPM per sample (Lange et al., 1997). FCM is frequently used in biomedical research to quantify eukaryotic cell populations and in microbiology to quantify a wide variety of yeast and bacterial cells (Nir et al., 1990; Van Dilla et al., 1983). FCM is also used to study environmental samples, e.g., to differentiate low nucleic acid (LNA) from high nucleic acid (HNA) phytoplankton in aquatic environments (Y. Wang et al., 2010; Müller and Nebe-von-Caron, 2010). Despite its advantages, FCM has seen little use in the bioaerosol field to date, owing in part to the challenges associated with collecting sufficient PBAP mass for robust counting statistics to be obtained (Chen and Li, 2005; Liang et al., 2013). Chen and $\mathrm{Li}$ (2005) determined that, for counting purposes, the SYTO-13 nucleic acid stain is the most effective (among five different nucleic acid stains studied) for determining reliable concentration of bioaerosols.

Light-induced fluorescence (LIF) is an increasingly utilized technique for bioaerosol quantification, and it relies on measuring the autofluorescence intensity of specific highyield fluorophores (e.g., nicotinamide adenine dinucleotide - NADH co-enzyme - flavins, and amino acids like tryptophan and tyrosine) present in PBAP. The major advantage of the technique is that it is fully automated, does not require a liquid suspension (i.e., it directly senses particles suspended in air), and provides high-frequency measurements $(\sim 1 \mathrm{~Hz})$, making it ideal for continuous monitoring and operation in highly variable environments (e.g., aircraft operation). Particles detected by LIF, called fluorescent biological aerosol particles (FBAP), although not equal to PBAP, may still constitute a large fraction of the biological particles (Healy et al., 2014; Gosselin et al., 2016). Using LIF, FBAP diurnal cycles showing maximum concentrations during evenings and minima around middays, especially in heavily vegetated environments, have been observed. This behavior has been related to known temperature and relative humidity release mechanisms of certain fungal spore species (Wu et al., 2007; Gabey et al., 2010; Tropak and Schnaiter, 2013). Huffman et al. (2010) used a Ultraviolet-Aerodynamic Particle Sizer (UV-APS) to show that the concentration and frequency of occurrence of $3 \mu \mathrm{m}$ FBAP particles at Mainz, Germany (semi-urban environment), exhibited a strong diurnal cycle from August through November, with a first peak at mid-morning (06:00-08:00; $1.6 \times 10^{4} \mathrm{~m}^{-3}$ ) followed by a constant profile $\left(\sim 2-4 \times 10^{4} \mathrm{~m}^{3}\right)$ throughout the rest of the day. Similar studies in urban and densely vegetated environments suggest a notable difference in the size distributions, diurnal behavior, and FBAP loading between the 
two environments. Gabey et al. (2011) found that the FBAP in Manchester, UK, follow a characteristic bimodal distribution with peaks at 1.2 and $1.5-3.0 \mu \mathrm{m}$. As in Mainz, the concentration of larger particles peaks in the mid-morning and ranges from 0 to $300 \mathrm{~L}^{-1}$, and the $1.2 \mu \mathrm{m}$ peak is linked to traffic activity. However, at the Borneo tropical rainforest FBAP concentrations peak during the evening with a robust 2-3 $\mu \mathrm{m}$ population and concentrations ranging from 100 to $2000 \mathrm{~L}^{-1}$ (Gabey et al., 2010).

LIF-based observations (e.g., UV-APS, WIBS), combined with measurements of molecular tracers (e.g., mannitol and arabitol) and endotoxin measurements, provide a more complete picture of PBAP emissions. Gosselin et al. (2016) applied this approach during the BEACHON-RoMBAS field campaign. A clear correlation between FBAP and the molecular markers is seen, indicating an increase in fungal spores during rain events. FBAP concentrations and molecular marker-inferred (arabitol and mannitol; Bauer et al., 2008a approach) fungal spore concentrations were within the same order of magnitude. The WIBS-3 cluster (determined using Crawford et al., 2015) linked to fungal spores gave concentrations $13 \%$ lower than those derived from molecular marker concentrations during rain events. During dry events, FBAP and molecular marker-derived fungal spore concentrations were poorly correlated. The degree to which all types of PBAP are consistently detected by LIF over different times of the year and different environments is currently unknown; it is likely, however, that for certain classes of bioparticles (e.g., pollen and fungi) the detection efficiency using LIF is relatively high. However, the low intrinsic fluorescence intensity of bacteria and high variability thereof in relation to metabolic state may lead to their misclassification as nonbiological particles (Hernandez et al., 2016).

For LIF-based quantification of PBAP to be effective, it requires the intrinsic fluorescence of biological material to exceed that of non-biological matter. Depending on the type, metabolic state, and species, PBAP autofluorescence may vary by orders of magnitude, and therefore LIF may not always be able to differentiate between biological and abiotic particles. For example, Tropak and Schnaiter (2013) showed that laboratory-generated mineral dust, soot, and ammonium sulfate may be misclassified as FBAP. To address misclassification, excitation emission matrices (EEMs) have been developed for biomolecules (e.g., tryptophan, tyrosine, riboflavin) and non-biological molecules (e.g., pyrene, naphthalene, humic acid). EEMs provide the wavelengthdependent fluorescence emission spectra as a function of the excitation wavelength and are used to assign spectral modes to known fluorophores. The structure of EEMs is important for identifying molecules that are unique to PBAP and allows their identification by LIF; it is this principle upon which detectors in commercial FBAP measurements (e.g., WIBS, UV-APS) are based. Comparison of EEMs from biological and non-biological molecules shows that even when biomolecules have higher autofluorescence intensity than non-biologicals in the LIF detection range, interferences from non-biological compounds (e.g., polycyclic aromatic hydrocarbons, and soot) from combustion emissions can influence LIF detection (Pöhlker et al., 2012). Considerable work remains on determining which detector(s) or combination thereof provides an unambiguous identification of bioaerosols and related subgroups (e.g., bacteria, fungal spores, pollen). Towards this, an aerobiology catalog of pure cultures has been developed for the WIBS-4, showing that instrument-to-instrument variability in fluorescence detection poses a considerable challenge, as applying common detection thresholds across instruments leads to considerable differences in PBAP concentration and composition (Hernandez et al., 2016).

Another important issue for LIF-based quantification of PBAP is the impact of atmospheric oxidants, UV, and other stressors on the fluorescence intensity of PBAP. Pan et al. (2014) tested the effect of relative humidity and ozone exposure on the autofluorescence spectra of octapeptide aerosol particles using an UV-APS connected to a rotating drum. Octapeptides, organic molecules containing eight amino acids and present in cells, were used as a proxy to study the aging of tryptophan, and results suggest that bioaerosol exposure to high but atmospherically relevant levels of ozone ( 150 ppb) decreases tryptophan fluorescence intensity and PBAP detection. Multiple stressors can affect bioaerosol LIF detection, so such issues need to be thoroughly explored to understand PBAP detection efficiency over the wide range of atmospheric conditions and PBAP population composition (Tropak and Schnaiter, 2013; Hernandez et al., 2016).

The aims of the study are to (i) develop an effective and reliable FCM detection and quantification protocol for bioaerosol; (ii) apply the protocol to understand bioaerosol populations and their variability during different meteorological conditions; and (iii) compare FCM and WIBS-4A results to have a better understanding of PBAP day-to-day variability. To our knowledge, this study is the first to develop a FCM protocol to identify and quantify well-defined speciated bioaerosol populations from samples collected from a modified state-of-the-art biosampler. LIF sampling of bioaerosol side by side with established and quantitative biology tools (FCM and EPM) was conducted to assess the LIF detection capabilities toward different bioaerosol populations and under atmospherically relevant conditions during this study. Atlanta is selected as a case study for PBAP sampling, as it provides a highly populated urban environment surrounded by vast vegetative areas; this and the broad range of temperature and humidity ensure a wide range of PBAP population composition, state, and concentrations. All the samples collected are compared side by side to concurrent WIBS-4A data collected over the same time period. 


\section{Instrumentation and methodology}

\subsection{Bioaerosol sampler}

Sampling was performed using the SpinCon II (InnovaPrep LLC, Inc.) portable wet-walled cyclone aerosol sampler. Aerosol is collected by inertial impaction with a recirculating liquid film in the cyclone; evaporative losses are compensated so that the sample volume is kept constant during a sample cycle. The particle collection efficiency for 1,3 , 3.5 , and $5.0 \mu \mathrm{m}$ particles is about $47.3 \pm 2.1 \%, 56.1 \pm 3.9 \%$, $14.6 \pm 0.6 \%$, and $13.8 \pm 2.2 \%$, respectively (Kesavan and Sagripanti, 2015). However, the experiments conducted using 1 and $3 \mu \mathrm{m}$ polystyrene latex (PSL) beads, $3.5 \mu \mathrm{m}$ oleic acid and $5.0 \mu \mathrm{m}$ oleic acid particles do not necessarily quantify the collection efficiency of biological particles in this size range. Even with a lower collection efficiency than any impingement sampler, SpinCon effectively collects larger amounts of biological particles owing to its high volumetric flow rate, which is a considerable advantage (Kesavan and Sagripanti, 2015). However, the stress caused by the high flow rate of the SpinCon may affect cell viability. ŠantlTemkiv et al. (2017) recently studied the SpinCon retention efficiency from seawater samples and for $P$. agglomerans populations from pure cultures $\left(\sim 10^{5}\right.$ cells $\left.\mathrm{mL}^{-1}\right)$. After $1 \mathrm{~h}$ of sampling, the SpinCon was found to retain $20.6 \pm 5.8 \%$ of the P. agglomerans concentration and $55.3 \pm 2.1 \%$ of the seawater microbial concentration.

In our study, the biosampler was run at $478 \mathrm{~L} \mathrm{~min}^{-1}$ for $4 \mathrm{~h}$ sampling cycles. Phosphate-buffered saline (PBS) $1 \mathrm{X} \mathrm{pH} 7.4$ solution was used and the instrument compensated for water evaporation by supplying Milli-Q water to keep the PBS concentration constant. Upon termination of each sampling cycle, the instrument was programmed to dispense the sample in a $15 \mathrm{~mL}$ centrifuge tube. Then, $10 \mu \mathrm{L}$ of formalin $(37 \mathrm{wt} \%$ formaldehyde) per $\mathrm{mL}$ of solution was added to every sample for preservation, and samples were stored at $4{ }^{\circ} \mathrm{C}$. Given the long sampling times and the low concentration of PBAP, the fluid supply system of the instrument was modified, and a cleaning protocol (CP) has been developed which is described below.

The SpinCon II water and PBS supply bags used in the commercial instrument were replaced by two $2 \mathrm{~L}$ autoclavable Nalgene bottles (Thermo Scientific Inc.) with antimicrobial tubing, connectors, and a small HEPA filter connected to vent and prevent coarse and submicron particle contamination (Fig. 1). Bottles were autoclaved and filled with Milli-Q water and PBS, beforehand sterilized with $0.2 \mu \mathrm{m}$ pore bottle top filters (Thermo Fisher Inc.), and transferred inside a biosafety cabinet. An aliquot of each fluid obtained after preparation was evaluated for sterility by EPM and FCM.

The cleaning protocol (CP) of the biosampling system consists of two phases. During phase one, all acrylic windows and the outside of the collector/concentrator were cleaned with ethanol $70 \mathrm{wt} \%$. Then, the instrument inlet and outlet and the inside of the collector/concentrator were cleaned with ethanol $70 \mathrm{wt} \%$. In the second phase, the SpinCon II inlet was connected to a HEPA filter to provide a particle-free source of air to the sampling system; the instrument was then washed with ethanol $70 \mathrm{wt} \%, 10 \mathrm{wt} \%$ bleach solution, PBS, and Milli-Q $\mathrm{H}_{2} \mathrm{O}$, respectively. The wash consisted of a rinse, a $2 \mathrm{~min}$ sample, and filling the instrument collector/concentrator with the fluid in use (i.e., bleach solution, ethanol, PBS, and Milli-Q $\mathrm{H}_{2} \mathrm{O}$ ). The collector/concentrator was drained after $1 \mathrm{~min}$. The above steps were repeated for the remaining fluids, taking $5 \mathrm{~min}$ per fluid. Overall, the $\mathrm{CP}$ requires $45 \mathrm{~min}$; upon completion, a blank is obtained to constrain the residual contamination levels after cleaning (described below). Finally, the HEPA filter was disconnected, instrument inlets and outlets were sealed, and the inlet tube was cleaned with ethanol $70 \mathrm{wt} \%$ to be ready for rooftop sampling. SpinCon II was rinsed with ethanol $70 \mathrm{wt} \%$ after each sampling episode and the cleaning protocol was applied before each sample.

Several blanks were obtained to quantify the levels of PBAP contamination in the fluids and sampler and to ensure that they were sufficiently low to not bias the detection, identification, and quantification of the PBAP. Furthermore, an instrument blank was obtained after a $\mathrm{CP}$ to constrain residual particles, by running the sampler for $2 \mathrm{~min}$, while sampling air with a HEPA filter connected to the inlet of the SpinCon II. Another blank was collected to characterize any contamination of biological particles from the supply of PBS and water in the SpinCon II. This was done by operating the SpinCon II for a $4 \mathrm{~h}$ period with a HEPA filter connected to the inlet which completely cleans the air entering the wet cyclone from any bioparticles. All blanks were analyzed directly via FCM (Sect. 2.3) and EPM.

The volumetric flow rate within the SpinCon II was routinely calibrated by a VT100 Hotwire Thermo-anemometer (Cole Palmer Inc.) using a three-hole round duct transverse approach. A $11 / 4 \mathrm{in}$. OD tube with the same diameter as the SpinCon II inlet was designed with three holes. Each hole was $60^{\circ}$ apart from the other and the holes were perpendicular to the axial air flow direction of the tube (Supplement, Fig. S1). Triplicates of flow rate measurements were taken in each hole at the center of the tube and averaged to determine the SpinCon II volumetric flow rate $\left(478.0 \pm 6.4 \mathrm{~L} \mathrm{~min}^{-1}\right)$.

\subsection{Flow cytometry}

During this study, a BD Accuri C6 flow cytometer (BD Bioscience Inc.) was used for flow cytometry. The instrument quantifies suspended cells in aqueous medium at three flow velocity modes (slow, medium, and fast flow at 14, 35, and $66 \mu \mathrm{L} \mathrm{min}^{-1}$, respectively). It excites particles with a $488 \mathrm{~nm}$ laser and possesses four fluorescence detectors, FL1 $(533 \pm 30 \mathrm{~nm})$, FL2 $(585 \pm 40 \mathrm{~nm})$, FL3 $(>670 \mathrm{~nm})$, and FL4 $(675 \pm 25 \mathrm{~nm})$, which make it possible to analyze the flu- 


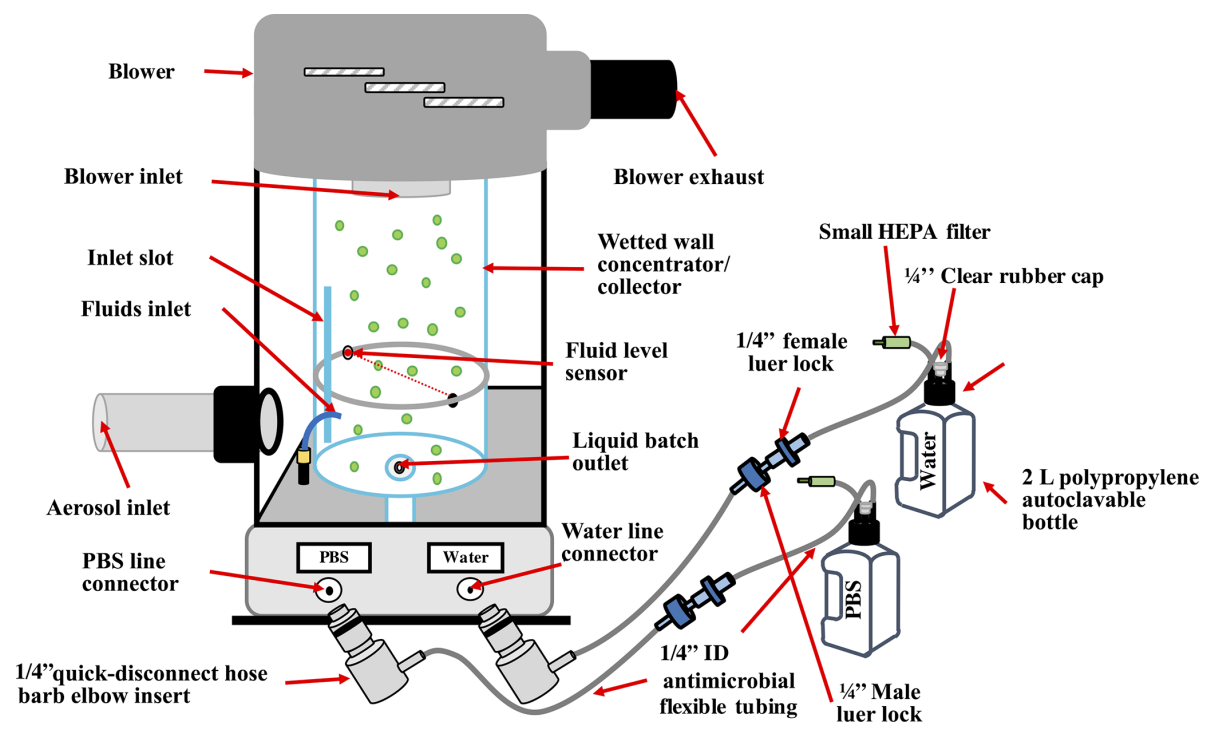

Figure 1. SpinCon II sampling setup including a modified fluid supply system with anti-microbial tubing and $2 \mathrm{~L}$ autoclavable bottles.

orescence from multiple dyes concurrently. In this study, a $2.5 \mu \mathrm{M}$ SYTO-13 nucleic acid probe was added to the fixed samples and incubated for $15 \mathrm{~min}$ in the dark at room temperature to stain biological particles. Additionally, $10 \mu \mathrm{L}$ of $15 \mu \mathrm{m}$ polystyrene bead suspension was added to the $1 \mathrm{~mL}$ total volume samples as an internal standard for PBAP concentration and size quantification. The BD Accuri C6 was cleansed before each use with $0.2 \mu \mathrm{m}$ filtered Milli-Q water in fast mode for $10 \mathrm{~min}$; background particle counts were typically reduced to $1 \mu \mathrm{L}^{-1}$. At the beginning of every experiment, a $1 \mathrm{~mL}$ blank of the atmospheric sample without SYTO-13 and beads was analyzed, used in quantification calculations (Sect. 3.1). Each sample was run in slow mode for $5 \mathrm{~min}$. After each sample, the instrument was flushed with $0.2 \mu \mathrm{m}$ filtered Milli-Q water in slow flow for $1 \mathrm{~min}$ (important for robust quantification of the typically low concentrations of the atmospheric samples). SYTO-13 fluorescence intensity was quantified by the FL1-A detector and used in combination with other parameters (FSC-A and SSC-A) to constrain the PBAP populations present. FSC-A measured forward $\left(0 \pm 13^{\circ}\right)$ scattering and is used to characterize the size of particles; SSC-A measured the side $\left(90 \pm 13^{\circ}\right)$ scattering and is used to characterize the internal complexity of particles. The SSC-A scattering intensity is a function of the cellular granularity or density of the internal structures (e.g., nucleus, mitochondria, ribosomes), the sphericity, and the size of the particles. Compared to spherical particles of the same size, elongated particles tend to yield a broader distribution of side-scattering intensities (Mage et al., 2019; Mathaes et al., 2013). Although side-scattering intensity increases with particle size, it has not been commonly used to measure cell size (Tzur et al., 2011). Overall, SSC-A scattering intensity will be proportional to the amount of scattering caused by the internal structures and the cell membrane, which ulti- mately depends on the refractive index of each cell (Müller and Neben-von-Caron, 2010). Side scattering has been effective at distinguishing cells of different complexities (e.g., monocytes and granulocytes; Shapiro, 2005).

An 80000 unit intensity FSC-H threshold (default FSC$\mathrm{H}$ threshold value suggested by the manufacturer to minimize the effect of noise) was set in the instrument during data acquisition to minimize the effects of noise on bioparticle counts. The FSC-H channel (where $\mathrm{H}$ denotes height) measures single-particle forward-scattering (FSC) intensity based on the peak (maximum point) of the voltage pulse curve recorded when a single particle goes through the interrogation point in the flow cytometer, whereas FSC-A, where A denotes area, measures single-particle FSC intensity based on the area below the curve of the recorder pulse. When the 80000 unit FSC-H threshold is defined, only signals with an intensity greater than or equal to the threshold value will be processed, and this could affect the statistics and detection efficiency of the flow cytometer toward small particles $(\leq 1 \mu \mathrm{m})$. Experiments conducted with $1.0 \mu \mathrm{m}$ polystyrene bead suspension (Supplement; Fig. S16) have shown that $1.0 \mu \mathrm{m}$ beads have FSC-H intensities above the $80 \mathrm{k}$ threshold, no particle losses are observed, and beads' estimated concentration agrees with that reported by the manufacturer $\left(\sim 6 \times 10^{7} \mathrm{~mL}^{-1}\right.$; Life Technologies, Inc.). The FCM data from each sample were analyzed using the FlowJo software (https://www.flowjo.com/solutions/flowjo, last access: 22 August 2018) to gate and quantify the bioparticle population. The same procedure was used to analyze the PBS, Milli-Q water, and blanks. 


\subsection{LIF detection of PBAP}

The WIBS-4A (referred to henceforth as "WIBS") is a single biological particle real-time sensor, which measures particle light scattering and autofluorescence in an approximately 0.5-15 $\mu \mathrm{m}$ particle range (http://www.dropletmeasurement. com, last access: 22 August 2018). Particles are initially sized using the $90^{\circ}$ side-scattering signal from a $635 \mathrm{~nm}$ continuous-wave diode laser. The scattering intensity is directly related to particle diameter and was calibrated prior to deployment using polystyrene latex sphere calibration standards (PSL with 0.8, 0.9, 1.0, 1.3, 2.0, and $3.0 \mu \mathrm{m}$ diameters, Thermo Scientific Inc.). The WIBS optical size therefore refers to PSL material with a real refractive index of 1.59. Healy et al. (2012) determined WIBS-4 counting efficiency by aerosolizing standardized concentrations of the PSL sphere of specific sizes (e.g., 0.3, 0.4, 0.56, 0.7, 0.9, and $1.3 \mu \mathrm{m}$ ) and compared WIBS-4 total counts against PSL counts detected by the condensation particle counter (CPC). Results show WIBS-4 possesses a 50\% counting efficiency for $0.5 \mu \mathrm{m}$ particles and detects $100 \%$ of the PSL particles above $0.7 \mu \mathrm{m}$ when it is compared to the CPC counts. The 280 and $370 \mathrm{~nm}$ pulsed Xenon flashtube UV lights in the WIBS cause the particles to autofluoresce (i.e., excite the chromophores preexisting in the PBAP and do not rely on a fluorescent dye as done in FCM). Then, fluorescent emissions are measured at three wavelength channels, which following the nomenclature of Perring et al. (2015) are (i) channel A ("FL1_280" in previous studies; Robinson et al., 2013), which refers to the detected emission between 310 and $400 \mathrm{~nm}$ after excitation at $280 \mathrm{~nm}$, (ii) channel B ("FL2_280" in previous studies), which refers to the detected emission between 420 and $650 \mathrm{~nm}$ after excitation at $280 \mathrm{~nm}$, and (iii) channel C ("FL2_370" in previous studies), which refers to the detected emission between 420 and $650 \mathrm{~nm}$ after excitation at $370 \mathrm{~nm}$. The resulting autofluorescence from $280 \mathrm{~nm}$ excitation is affected by the presence of tryptophan, tyrosine, and phenylalanine amino acids in the PBAP (Pöhlker et al., 2012). Similarly, the resulting autofluorescence from the $370 \mathrm{~nm}$ excitation is influenced by the presence of riboflavin and co-enzyme nicotinamide adenine dinucleotide phosphate $(\mathrm{NAD}(\mathrm{P}) \mathrm{H})$ within the cells.

Biological and non-biological particles can be discriminated by using a fluorescent intensity threshold; here the threshold is determined with the Gabey et al. (2010) method and with modifications by Perring et al. (2015) as follows. Particles with fluorescence intensities below the fluorescence threshold in all channels are categorized as non-fluorescent (NON-FBAP). Particles that fluoresce above the threshold in only one channel are named with a single letter (e.g., A, B, or C); particles that fluoresce in two channels are named with the two channel letters (e.g., $\mathrm{AB}, \mathrm{AC}$, or $\mathrm{BC}$ ), while particles that fluoresce in all channels are categorized as type ABC. Furthermore, the total FBAP concentration is defined as the sum of the concentration in the seven FBAP categories defined above. This approach was applied by Hernandez et al. (2016) to pure culture PBAP (bacteria, fungal spores, pollen) to study their correspondence to FBAP types; bacteria tend to be detected by type $\mathrm{A}$ and fungal spores and pollen by types $\mathrm{AB}$ and $\mathrm{ABC}$. However, bioaerosol classification is instrument-specific and particle-size-dependent (Hernandez et al., 2016; Savage et al., 2017). Multiple environments have been studied using the Perring et al. (2015) FBAP types, including rural, urban, and highly vegetated locations. In the southeastern US, the total FBAP concentrations range from $2 \times 10^{4}$ to $8 \times 10^{4} \mathrm{~m}^{-3}$, constituting $3 \%-24 \%$ of the total supermicron particle number between 1 and $10 \mu \mathrm{m}$ diameter. In the highly vegetated Rocky Mountains, ABC-type particles are enhanced during rainy days (during or post rain events) to $\sim 65 \%$ of the total FBAP, owing to the release of wet-ejected fungal spores following precipitation (Gosselin et al., 2016). By contrast, in the highly populated city of Nanjing, China, all FBAP types, except type C, correlated with black carbon concentrations, suggesting a strong interference by combustion sources (Yu et al., 2016). A detailed explanation of the above-mentioned studies using the Perring et al. (2015) approach is also included in Sect. S20 of the Supplement.

\subsection{Location of sampling site and sampling frequency}

Bioaerosol sampling was conducted between 7 April and 15 May 2015 at the rooftop sampling platform of the Ford Environmental Sciences and Technology (ES\&T) building at the Georgia Institute of Technology campus in Atlanta, GA. The site, which was located at the heart of a major urban environment, is surrounded by densely forested areas in the southeastern USA: the Oconee National Forest (southeast), the Chattahoochee National Forest (north), and the Talladega National Forest (west). The WIBS operated continuously throughout the same period, sampling bioaerosol from a $15 \mathrm{ft}(\sim 4.6 \mathrm{~m})$ long and $1 / 4 \mathrm{in}$. ID conductive tubing inlet fixed $8 \mathrm{ft}(\sim 2.4 \mathrm{~m})$ above the sampling platform floor. The SpinCon II was placed in the platform during sampling episodes with its inlet facing south. Three $4 \mathrm{~h}$ samples per week were collected with the Spincon II sampler over the 5-week period (4h sampling between 10:00 and 17:00; Table 1). Meteorological data acquired from the same platform provided wind speed, wind direction, relative humidity (RH), temperature, total hourly rain, and UV radiation index with a 1 min resolution.

\section{Data processing and analysis}

\subsection{FCM data processing}

All blanks collected showed contamination levels that did not exceed $1 \%$ of the PBAP quantified in the subsequent atmospheric samples. The $2 \mathrm{~min}$ instrument blanks obtained after the $\mathrm{CP}$ and the HEPA filter washes were $1.06 \times 10^{3} \pm 7.37 \times$ $10^{2}$ and $9.22 \times 10^{2} \pm 1.24 \times 10^{2} \mathrm{~mL}^{-1}$, respectively, which are 
Table 1. Summary of the SpinCon II sampling events, the $24 \mathrm{~h}$ averaged $\mathrm{RH}$, ambient temperature, the assigned meteorological category (using Sect. 4.4 definitions), and the corrected FCM-derived PBAP number concentration (1 to $5 \mu \mathrm{m}$ ) for each sample collected during this study.

\begin{tabular}{lrrlr}
\hline $\begin{array}{l}\text { Date } \\
\text { (starting-ending time) }\end{array}$ & $\begin{array}{l}\text { RH } \\
(\%)\end{array}$ & $\begin{array}{r}\text { Temperature } \\
\left({ }^{\circ} \mathrm{C}\right)\end{array}$ & $\begin{array}{l}\text { Meteorological } \\
\text { category }\end{array}$ & $\begin{array}{r}\text { PBAP concentration }\left(\mathrm{m}^{-3}\right) \\
1 \text { to } 5 \mu \mathrm{m} \text { diameter range }\end{array}$ \\
\hline 7 April 2015 $(11: 17-15: 17)^{\mathrm{a}}$ & 70.9 & 21.4 & Humid, warm & $9.282 \times 10^{4}$ \\
8 April 2015 $(11: 10-15: 10)$ & 53.6 & 24.9 & Dry, warm & $5.203 \times 10^{5}$ \\
9 April 2015 $(11: 15-15: 15)$ & 53.8 & 25.3 & Dry, warm & $1.254 \times 10^{5}$ \\
14 April 2015 $(11: 30-15: 30)^{\mathrm{a}}$ & 76.8 & 22.5 & Humid, warm & $8.253 \times 10^{4}$ \\
15 April 2015 $(11: 40-15: 40)^{\mathrm{a}}$ & 83.6 & 18.9 & Humid, warm & $1.234 \times 10^{5}$ \\
16 April 2015 $(10: 55-14: 55)$ & 86.3 & 12.5 & Humid, cold & $3.399 \times 10^{5}$ \\
21 April 2015 $(13: 15-17: 15)$ & 43.2 & 16.6 & Dry, cold & $4.741 \times 10^{5}$ \\
22 April 2015 $(11: 25-15: 25)$ & 38.1 & 18.8 & Dry, warm & $3.351 \times 10^{5}$ \\
23 April 2015 $(11: 35-15: 35)$ & 48.1 & 16.8 & Dry, cold & $1.708 \times 10^{6}$ \\
28 April 2015 $(12: 25-16: 25)$ & 45.3 & 17.0 & Dry, cold & $4.899 \times 10^{5}$ \\
29 April 2015 $(11: 55-15: 55)^{\mathrm{b}}$ & 79.4 & 14.2 & Humid, cold & $4.591 \times 10^{5}$ \\
30 April 2015 $(12: 10-16: 10)$ & 57.3 & 17.4 & Dry, cold & $9.603 \times 10^{5}$ \\
13 May 2015 $(10: 50-14: 50)$ & 40.1 & 23.5 & Dry, warm & $3.680 \times 10^{5}$ \\
14 May 2015 $(11: 50-15: 50)$ & 52.3 & 23.0 & Dry, warm & $4.851 \times 10^{5}$ \\
15 May 2015 $(10: 19-14: 19)$ & 64.4 & 23.1 & Dry, warm & $1.656 \times 10^{6}$ \\
\hline
\end{tabular}

${ }^{\mathrm{a}}$ Sampling occurred post rain event. ${ }^{\mathrm{b}}$ Sampling occurred during a rain event.

negligible accumulations compared to the $2.55 \times 10^{5} \pm 1.14 \times$ $10^{5} \mathrm{~mL}^{-1}$ average PBAP concentration quantified in the atmospheric samples. The concentration of PBAP in the blanks was also confirmed with microscopy (not shown). Based on this, we are confident that the $\mathrm{CP}$ protocol and procedure to replace the working fluids ensured sterility of the biosampler before each sampling.

FCM analysis of the samples was carried out as follows. We obtain the fluorescence intensity (from each of the four fluorescence detectors) and forward-scattering and side-scattering intensity for all the particles suspended in the samples. A gating procedure was used to determine the fluorescence levels associated with detecting only particles containing SYTO-13 (hence, a PBAP) and background fluorescence from non-stained particles. The procedure (Supplement, Sects. 2 and 3) consists of three steps: (a) fluorescence threshold determination, (b) population gating, and (c) biological/non-biological particle discrimination in the population(s) within the threshold (e.g., LNA PBAP, Sect. 4.1). The fluorescence threshold was determined using an atmospheric sample without SYTO-13 collected before each FCM analysis, as a blank. Based on the fluorescence responses obtained, we determine the FL1-A fluorescence intensity value for which $99.5 \%$ or $99.9 \%$ of the (unstained) particles of the blank autofluoresce below the chosen value. This FL1-A intensity, called the "fluorescence threshold", was determined for each sample (Supplement, Fig. S2a and b). The determination of the fluorescence threshold involved selecting the most conservative value that maximizes the inclusion of biological particles and minimizes the inclusion of non-biological particles, including those that may be subject to background fluorescence or unspecific binding of SYTO13 (Díaz et al., 2010; Müller and Nebe-von-Caron, 2010). We found out that threshold values for the $99.9 \%$ approach were substantially higher than the $99.5 \%$ approach in multiple sampling events and comparable to the fluorescence intensities observed for stained pure cultures $\left(\sim 10^{5}\right.$ units), which means that the $99.9 \%$ threshold values will miscount pure cultures as non-biological. Consequently, we set the fluorescence threshold to the highest fluorescence intensity value observed by the $99.5 \%$ approach (41 839 units; Supplement, Fig. S2b) and applied it to all collected samples, henceforth named the $42 \mathrm{k}$ FL1-A threshold. The $42 \mathrm{k}$ threshold value aims to minimize any abiotic interference as it maximizes biological particle quantification. A fixed value has been chosen and applied to all samples given that having a different threshold value for each sampling event may result in quantification biases as bioaerosols with strong autofluorescence (e.g., pollen, fungal spores) can increase the threshold value and affect PBAP quantification in the population(s) within the threshold. The BD Accuri C6 flow cytometer used for the analysis of the samples maintains constant pre-optimized photomultiplier voltages and amplifier gain settings. As a result, the fluorescence intensity of particles is consistent from day to day, and the fluorescence intensity of a specific biological particle population with the same metabolic state and physiological characteristics must not show day-today variability (http://www.bdbiosciences.com, last access: 9 August 2018). Under the $42 \mathrm{k}$ threshold approach PBAP concentrations in the population(s) within the threshold (e.g., 
LNA, Sect. 4.1) can be overestimated by up to $0.5 \%$. Furthermore, FCM experiments conducted with unprocessed Arizona Test Dust (ATD) show that the FL1-A intensity distribution of ATD particles stained with SYTO-13 is very similar to unstained ATD particles, and $100 \%$ of the SYTO-13stained ATD particles stay below the $42 \mathrm{k}$ threshold (Supplement, Fig. S14a and b), supporting the $42 \mathrm{k}$ threshold effectiveness in filtering out abiotic particles.

Once the FL1-A threshold was determined, plots of FL1-A vs. SSC-A and FL1-A vs. FSC-A were used to define clusters of bioparticles with fluorescence that exceed the FL1-A threshold and a characteristic optical size (obtained from the FSC-A intensity) or particle internal complexity (obtained from the SSC-A intensity). FL1-A vs. SSC-A plots were used to define the populations of bioparticles for PBAP quantification as clusters using the SSC-A parameter were more defined and showed better spatial resolution than when using the FSC-A parameter. The limits of each population were also determined with FlowJo (https://www.flowjo.com, last access: 22 August 2018), using $2 \%$ contour plots (Supplement Fig. S3) generated by equal probability contouring (i.e., 50 contour levels so that the same number of cells fall between each pair of contour lines). Populations above the FL1A threshold value (41 839 FL1-A units) were considered biological (Sect. 4.1; e.g., HNA); the particles in the population within the threshold value (Sect. 4.1; e.g., LNA) with a FL1A intensity greater than 41839 units were counted as biological to determine the PBAP counts in the population. The total PBAP counts were considered to be all particle counts with FL1-A fluorescence intensity above the determined threshold value minus the $15 \mu \mathrm{m}$ beads' internal standard with FL1-A fluorescence intensity above the determined threshold value. The $15 \mu \mathrm{m}$ beads of known concentration and particle size allow for calibration of the optical size (Supplement Sect. S7) of the bioparticles as well as their concentration and departure from sphericity. The $15 \mu \mathrm{m}$ bead population showed fluorescence intensities comparable to the determined fluorescence threshold after been stained with SYTO-13, as it is known that molecular stains can be adsorbed on the surface of polystyrene beads (Eckenrode et al., 2005; Rödiger et al., 2011). The relatively high fluorescence intensity of the $15 \mu \mathrm{m}$ beads shows that populations within the threshold value (e.g., LNA, Sect. 4.1) cannot be ruled out as being affected by unspecific staining of abiotic particles. However, populations above the threshold value (e.g., HNA, Sect. 4.1) should not be affected by such abiotic interferences.

\subsection{WIBS data processing}

Fifteen minute average total aerosol and FBAP size distributions were obtained from the WIBS. FBAP was distinguished from the total aerosol using the Gabey et al. (2010) "trigger threshold" approach, which is applied as follows. First, the average "electronic fluorescence noise" and its standard deviation are determined for each channel $(\mathrm{A}, \mathrm{B}, \mathrm{C})$ by per- forming the force trigger (FT) calibration which consists in operating the WIBS without flowing air through the system. The FT calibration, carried out every $24 \mathrm{~h}$, is critical for determining the lowest particle autofluorescence levels that robustly exceed instrument electronic noise. FT calibrations measured the particle-free air background autofluorescence in the three WIBS channels (e.g., A, B, C), and measurements recorded the fluorescence intensity for 500 excitation flash events (Ziemba et al., 2016; Tropak and Schnaiter, 2013; Gabey et al., 2010). The threshold for each detector is then equal to the average fluorescence plus 2.5 times its standard deviation; particles with fluorescence intensities above this threshold value are classified as FBAP. Then, the Perring et al. (2015) approach (Sect. 2.3) is applied to determine the combination of thresholds that provide the maximum concentration of PBAP and minimal interference from abiotic particles, which still remains an area of active research. It is important to note that the Gabey et al. (2010) threshold approach and the Perring et al. (2015) FBAP types were applied to the WIBS-4A data and should not be directly compared to FBAP quantifications performed by the WIBS-3 in previous studies, owing to the channel A and B overlap on the latter. A detailed comparison between the WIBS- 3 and WIBS- 4 models, as well as PBAP detection by both models, is further discussed in the Supplement (Sect. S21).

In this study, thresholds for each channel were determined daily, and the total particle concentration, FBAP type (e.g., $\mathrm{A}, \mathrm{B}, \mathrm{C}, \mathrm{AB}, \mathrm{BC}, \mathrm{AC}$, and $\mathrm{ABC}$ ) concentrations, and total FBAP concentration (sum of the seven FBAP types) were used. From the data, $4 \mathrm{~h}$ averaged size distributions (using $15 \mathrm{~min}$ average data) were generated for the total particles and all FBAP types in the 1-10 $\mu \mathrm{m}$ range during the SpinCon II run. Subsequently, WIBS overall sampling efficiency (aspiration efficiency + transport efficiency) was calculated using the Particle Losses Calculator (Von der Weiden et al., 2009) and applied to the $1-10 \mu \mathrm{m}$ size distributions for the sampling characteristics in our setup $(15 \mathrm{ft}$ $(\sim 4.6 \mathrm{~m})$ sampling line with $1 / 4 \mathrm{in}$. ID and $2.3 \mathrm{~L} \mathrm{~min}^{-1}$ flow rate; Fig. S4a). The sampling efficiency was calculated to be $67 \%$ for $5 \mu \mathrm{m}$ particles, with larger losses as size increased to $10 \mu \mathrm{m}$ (Supplement, Fig. S4b). FCM and WIBS total particles and PBAP comparison was constrained to the 1 to $5 \mu \mathrm{m}$ range being the size overlap of both techniques. Also, the fractional composition of FBAP (based on number concentrations) was calculated to characterize its daily variability (Sect. 4.2), and compared against the daily variability of PBAP from the FCM analysis (Sect. 4.4). 


\section{Results and discussion}

\subsection{FCM biopopulation identification and quantification}

When the FCM results are plotted in terms of FL1-A fluorescence intensity vs. SSC-A scattering intensity, four populations (Fig. 2) emerge above the detection thresholds: LNA particles, HNA particles, pollen, and the $15 \mu \mathrm{m}$ internal standard beads. EPM and SEM pictures (Supplement Figs. S5, S6, and S7) confirm the presence of these heterogeneous populations. SYTO-13 stains DNA and RNA, and the resulting single-cell FL1-A fluorescence intensity (Fig. 2) is directly proportional to its nucleic acid content (Lebaron et al., 2001; Troussellier et al., 1999; Comas-Riu and Vives-Rego, 2002). Previously, SYTO-13 has effectively distinguish between HNA and LNA bacterioplankton and phytoplankton populations in fresh and seawater samples, and results are comparable to SYBR green II and SYBR green I, more specific DNA probes (Wang et al., 2010; Bouvier et al., 2007; Lebaron et al., 2001). However, corresponding populations in atmospheric PBAP have not been identified before. The SSC-A scattering intensity in Fig. 2 changes as a function of the size, composition (e.g., cell refractive index), and complexity of the cell (e.g., internal structures or surface irregularities) and the strongest SSC-A intensity corresponding to the largest, most complex particles. Below we focus on each population to further understand the identified populations of biological particles.

The HNA size distributions are dominated by $3-5 \mu \mathrm{m}$ particles (mean diameter: $4.15 \pm 0.06 \mu \mathrm{m}$; Supplement Fig. S10) and the total concentration moderately correlated with RH. HNA were virtually non-existent during several extended dry periods (days with average $\mathrm{RH}<70 \%$ during sampling, e.g., 9 April, 22 April, and 15 May) and well defined during periods of high humidity, especially after rain events (days with average $\mathrm{RH}>70 \%$ and $T>18{ }^{\circ} \mathrm{C}$ during sampling episode; e.g., 7, 14, and 15 April). Both of these characteristics suggest that HNA particles correspond to wet-ejected fungal spores (e.g., from the Ascospores and Basidiospores genus; Oliveira et al., 2009; Li and Kendrick, 1995). The LNA size distributions are dominated by $2-4 \mu \mathrm{m}$ particles (mean diameter: $2.99 \pm 0.06 \mu \mathrm{m}$; Supplement, Table S1) and dominated Atlanta PBAP composition during dry days. Many individual bacteria are likely in around $1 \mu \mathrm{m}$, but the observed LNA particles are within the median aerodynamic diameter of culturable bacteria $(\sim 4 \mu \mathrm{m})$ in continental sites (Després et al., 2012). Bacteria in the atmosphere can be co-emitted together with larger particles (e.g., soil, plant fragments), and occasionally they are observed as clumps of bacteria cells (Burrows et al., 2009). In addition, several bacterial species observed in the atmosphere (Delort and Amato, 2018; Monier and Lindow, 2003; Baillie and Read, 2001) are within this sizes range (e.g., Sphingomonas spp.: 1.0-2.7 $\mu \mathrm{m}$; Methylobacterium spp.: $1-8 \mu \mathrm{m}$, Pseudomona syringae: $\sim 2.5 \mu \mathrm{m}$,

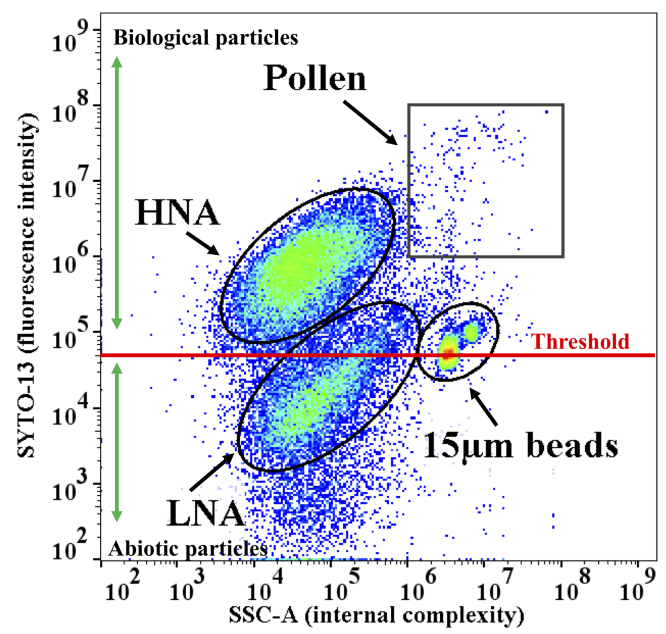

Figure 2. FL1-A vs. SSC-A plot used to identify populations in the 14 April 2015 sample, including the $42 \mathrm{k}$ threshold line in red and abiotic particle (below threshold) and biological particle (above threshold) designated regions. In the density plot green and red zones denote the most populated regions. FL1-A on the $y$ axis shows the fluorescence intensity of each particle in the plot stained with SYTO-13, and SSC-A on the $x$ axis measures $90^{\circ}$ light scattering related to the internal complexity (e.g., granularity or number of internal structures) of the particles. The fraction of the LNA population above the threshold line is referred to as the "LNA-AT" population.

and Bacillus anthracis: 3-10 $\mu \mathrm{m}$ ), supporting LNA population may represent single or agglomerated bacterial cells. However, it is clear that heterogeneous populations will probably contain multiple types of microorganisms, and that may be the case in the LNA population.

It is known that pollen may burst into tiny fragments when is suspended in water (e.g., Augustin et al., 2013; Taylor et al., 2007), potentially increasing the concentration of LNA particles and biasing concentrations. Although 0.2$5 \mu \mathrm{m}$ pollen fragments can be generated upon rupture, pollen (e.g., birch, ryegrass, oak, olive) mainly breaks apart into submicron fragments by hydrolysis and favors fragmentation into small submicron $(<1 \mu \mathrm{m})$ particles (Taylor et al., 2007; Bacsi et al., 2006; Grote et al., 2003) not considered in our FCM analysis. An additional factor to consider in pollen fragmentation is the number of fragments generated per pollen grain. FCM applied to ragweed pollen suggests a 1:2 pollen-to-pollen fragment concentration ratio (Supplement, Table S2). Also, calculations based upon FCMderived ragweed pollen and pollen fragments concentrations during this study (considering the total pollen mass added to the sample, the $15 \mu \mathrm{m}$ mean diameter previously determined by Lin et al., 2013, and unit density) suggest that approximately $67 \%$ of the ragweed pollen grains were intact after hydration and that each fragmented grain generates $\sim 5$ pollen fragments. Results agree with Bacsi et al. (2006) observations, where $35 \%$ of the ragweed pollen grains frag- 
ment upon hydration. Overall, ragweed pollen results suggest that FCM experiments do not have a considerable impact on pollen fragmentation and that pollen fragmentation will have a negligible effect on LNA concentrations. Ragweed pollen is one of the most abundant wind-driven pollen species in the United States and its emission peaks during fall, but can be also present during late spring and summer. It is representative of the pollen species we see in the Atlanta area (Darrow et al., 2012), and results suggest that pollen fragmentation would not generate a substantial number of fragments. The low collection efficiency of SpinCon toward large particles $(<14 \%$ for diameters above $5 \mu \mathrm{m})$ and that pollen concentrations in our samples are generally 2 orders of magnitude lower than LNA concentrations (Fig. S22; Supplement) suggest a negligible effect of pollen fragments on LNA biological particle quantification. Pollen concentrations are 100-1000 times lower than bacteria concentrations in the atmosphere (Hoose et al., 2010). At least 100 supermicron $(>1 \mu \mathrm{m})$ pollen fragments will have to be released per pollen grain to considerably influence the LNA population, which has not been observed. Also, EPM results showed intact pollen and limited amounts of small debris among the particles identified in the atmospheric samples collected for this study. Particles with fluorescence intensities above the FL1-A threshold value in the LNA population were counted as biological, giving us the PBAP counts within the LNA population, and will be referred to henceforth as the "LNAAT" population (Fig. 2), where "AT" refers to the above threshold.

The LNA population shows SYTO-13 fluorescence intensities that are about 1 order of magnitude lower than the HNA population, and the fluorescence intensity difference is consistent across all sampling events. Based on Bouvier et al. (2007), cell populations with different metabolic activity (e.g., active and non-active), when detected by FCM, should observe a decrease in fluorescence intensity in consecutive sampling events when transitioning from the HNA to the LNA population or vice versa when transitioning from the LNA to HNA population. The fluorescence intensity of the LNA and HNA populations shows small variation throughout the sampling events (LNA-AT: $7.38 \times 10^{4} \pm 1.39 \times 10^{4}$; HNA: $6.72 \times 10^{5} \pm 2.30 \times 10^{5}$; Table S3), and no anticorrelation is observed in the studied parameters (FSC-A, SSC-A, FL1-A), which suggests that we have in fact two distinctive populations of bioaerosols (Supplement; Figs. S23 and S15).

A population of strongly fluorescing and very large particles $(10-20 \mu \mathrm{m}$, average geometric mean diameter $12.3 \pm$ $1.7 \mu \mathrm{m}$ ) was identified (Fig. 2). This population also strongly autofluoresces in the FCM when SYTO-13 was not added to the sample (Sect. 8, Fig. S11). All together this indicates a population of pollen particles, as they are known to contain cell wall compounds (i.e., phenolic compounds, carotenoid pigments, Phenylcoumarin) that fluoresce more strongly than the proteins and cytosolic compounds responsible for bacteria/fungi autofluorescence (Pöhlker et al., 2012,
2013; Hill et al., 2009). The pollen population was not well defined during all sampling events. Whenever present, pollen was characterized by concentrations $\left(\sim 10^{2} \mathrm{~m}^{-3}\right)$ consistent with reported values (Després et al., 2012), which are also much lower than LNA-AT and HNA concentrations. As a result, pollen population was systematically gated using a perfect square between $10^{6}$ and $10^{8}$ intensity units in the FL1-A vs. SSC-A plot for each atmospheric sample. LNAAT, HNA, and pollen counts acquired by the $42 \mathrm{k}$ threshold approach were used to calculate liquid-based $\left(\mathrm{mL}^{-1}\right.$ of sample solution) and air-based $\left(\mathrm{m}^{-3}\right.$ of air) concentrations for each bioaerosol population as detailed in the Supplement. The total PBAP concentration on each sample consisted of all non-bead particles above the $42 \mathrm{k}$ fluorescence threshold given that a non-negligible biological particle concentration was not constrained in the gated populations. Even though the $2 \%$ contour plots effectively allowed population gating, $16.5 \pm 7.3 \%$ of the total PBAP are not attributed to the identified populations. The biological particles not constrained by FlowJo $2 \%$ gating, henceforth named the "unclassified" bioparticles, showed the highest concentrations when both HNA and LNA populations are densely populated (16 April, 28 April, and 14 May; Fig. 5). The lowest concentrations were observed when just the LNA population is identified (9 April, 22 April, 15 May; Fig. 5) and when the LNA and HNA populations are identified after the rain event on 14 April. The observed behavior shows that the unclassified bioparticle concentrations is linked to the heterogeneity of the biological populations and the concentration of the gated populations (e.g., HNA, LNA, and pollen). The "unclassified" bioparticle concentration ranges from $8.1 \times 10^{2}$ to $1.3 \times 10^{4} \mathrm{~m}^{-3}$ (average $4.2 \times 10^{3} \pm 3.3 \times 10^{3}$ ), and they are not constrained to a specific size range. Most of the unclassified bioparticles are far from the centroids of the gated populations. They can indeed be formed by fragmentation or accretion, or also be related to plant debris (i.e., irregular bioparticles) that are characterized by a very broad size, internal complexity and nucleic acid content distributions. In addition, we must note that additional concentration corrections are required owing to the sampling efficiency of the SpinCon II, but will be considered in Sect. 4.3 and 4.4.

Before SpinCon II sampling efficiency corrections are applied, FCM total particle concentrations range from $2.6 \times 10^{4}$ to $2.9 \times 10^{5} \mathrm{~m}^{-3}$, with increasing concentrations toward the end of the sampling period. In addition, total PBAP concentration averaged $2.4 \times 10^{4} \pm 1.1 \times 10^{4} \mathrm{~m}^{-3}$ (coefficient of variation, $\mathrm{CV}, 13 \%$; defined as the standard deviation over a triplicate FCM measurements over the average concentration). LNA-AT ranged between $6.8 \times 10^{2}$ and $2.9 \times$ $10^{4} \mathrm{~m}^{-3}$ (average: $1.1 \times 10^{4} \mathrm{~m}^{-3}$; CV: $20 \%$ ), HNA (fungal spores) between $4.7 \times 10^{3}$ and $1.9 \times 10^{4} \mathrm{~m}^{-3}$ (average: $\left.1.1 \times 10^{4} \mathrm{~m}^{-3} ; \mathrm{CV}: 15 \%\right)$ when above the detection limit $(n=12)$, and pollen from $1.3 \times 10^{2}$ to $1.2 \times 10^{3} \mathrm{~m}^{-3}$ (average: $3.6 \times 10^{2} \mathrm{~m}^{-3} ; \mathrm{CV}: 21 \%$ ). These concentration levels are consistent with microscopy-based studies in urban en- 
vironments for bacteria (e.g., $1.7 \times 10^{4} \pm 1.3 \times 10^{4} \mathrm{~m}^{-3}$ in springtime Birmingham, UK; Harrison et al., 2005); fungal spores $\left(1.8 \times 10^{4} \pm 1.1 \times 10^{4} \mathrm{~m}^{-3}\right.$ in Vienna, Austria, between April and June; Bauer et al., 2008b); and pollen (between $5.69 \times 10^{2} \mathrm{~m}^{-3}$ and $6.144 \times 10^{3} \mathrm{~m}^{-3}$ in Medellin, Colombia; Guarín et al., 2015). Also, additional experiments performed in September 2015, described in Fig. S7 of the Supplement (Sect. 6), showed that EPM and FCM-based quantifications agree within an order of magnitude. This is consistent with Lange et al. (1997), who also found that FCM gives higher quantifications than EPM microscopy when studying $P$. aeruginosa pure cultures and airborne bacteria collected from a swine confinement building in Iowa, USA.

To better understand SYTO-13 fluorescence intensity differences between the identified (e.g., LNA-AT, HNA, and pollen) populations in the atmospheric samples and their metabolic/stress state, FCM experiments were conducted with air-isolated bacteria (F8 strain; DeLeon-Rodriguez, 2015), ragweed pollen, and yeast (S. cerevisiae; Y55 strain) mixtures to compare the SYTO-13 fluorescence intensity and the scattering properties of the pure cultures to those seen in the atmospheric samples. Pure culture experiments aimed to (1) serve as positive controls to ensure SYTO-13 effectively stains bacteria, fungi, and pollen and (2) acquire reference fluorescence and scattering properties on each pure culture population. Pure cultures and atmospheric samples are summarized in Tables S3 and S4 (Supplement; FCM pure culture experiments), respectively. The LNA-AT population showed SYTO-13 fluorescence intensity up to 2 orders of magnitude lower than F8 bacteria. The HNA population showed an order of magnitude lower SYTO-13 fluorescence intensity than Y55 HNA yeast and within the same magnitude for the LNA Y55 yeast. The HNA and LNA yeast populations in the pure culture experiments (Fig. S13a) have 1 order of magnitude difference in FL1-A fluorescence intensity and may represent yeast populations with different metabolic states. Atmospheric and ragweed pollen populations had similar SYTO13 fluorescence intensities and Fig. S13c shows pollen fluorescence intensity may go up to $10^{8}$. The lower SYTO-13 fluorescence intensity of the atmospheric populations may be related to genetic material degradation from exposure to atmospheric stressors; depending on the physiological characteristics of each population (Zhen et al., 2013; Amato et al., 2015). Our results also agree with Guindulain et al. (1997), showing that $E$. coli overnight cultures have higher SYTO-13 fluorescence intensity than starved E. coli populations. Overall, FCM pure culture results suggest that microbes starve in the atmosphere, leading to a possible reduction or leakage of the amount genetic material enclosed within each cell. Sampling can also stress cells, even disrupt the wall/membrane of the cell, and lead to genetic material leakage (Zhen et al., 2013).

Pollen, HNA, and LNA-AT atmospheric populations showed different SYTO-13 fluorescence intensities. Pollen showed the highest fluorescence intensity, followed by the
HNA and LNA-AT (fraction of LNA above threshold; Fig. 2) populations, respectively (Fig. 2; Table S4). Guindulain et al. (1997) FCM results with starved bacterioplankton from seawater samples treated with DNase/RNase showed SYTO13 fluorescence intensity can be related to the DNA content of starved bacterioplankton due to the low amount of RNA enclosed in starved cells. Taking into consideration our results and previous studies, we can suggest that pollen, LNA-AT, and HNA populations in the atmospheric samples are distinguished by their DNA content, which can in part explain the SYTO-13 fluorescence intensity difference between them. We also acknowledge that DNA sequestration by bacteria, fungal spores, and pollen may differ, and their cell membrane characteristics will ultimately determine how much stress the cells will sustain before they completely rupture. SYTO-13 is a highly permeable stain and effectively detects nucleic acids (DNA and RNA) of bacteria endospores and vegetative cells (Comas-Riu and Vives-Rego, 2002). Fungal spores have also been effectively stained by DNA/RNA probes (Bochdansky et al., 2016; Chen and Li et al., 2005), but some fungal spores might not be equally stained due to their harder cell wall and chromatin binding of DNA (Standaert-Vitse et al., 2015). Future work is needed to study this further.

\subsection{WIBS total concentration and FBAP daily variability}

WIBS-4A collected data continuously throughout the period; for comparison against the SpinCon II $4 \mathrm{~h}$ liquid batch samples, WIBS data were averaged to the SpinCon II sampling times (Table 1). WIBS total particle concentration (1-5 $\mu \mathrm{m}$ diameter) ranged from $2.0 \times 10^{5}$ to $1.0 \times 10^{6} \mathrm{~m}^{-3}$, in agreement with observed particle concentrations in previously studied urban environments during spring/summer months like Helsinki, Finland (UV-APS average $1.6 \times 10^{5} \mathrm{~m}^{-3}$; Saari et al., 2014), and Karlsruhe, Germany (WIBS-4 average $6.9 \times 10^{5} \mathrm{~m}^{-3}$; Tropak and Schnaiter, 2013); $4 \mathrm{~h}$ average total particle concentrations in Fig. 3a show particle concentrations declined during rain episodes (during or post rain: e.g., $15,16,28,29$, and 30 April) as wet removal of PBAP is most efficient. However, during dry (no rain) episodes total particle concentrations built up in the atmosphere. To better understand the day-to-day variability of different FBAP types, the seven Perring et al. (2015) FBAP categories (i.e., types A, $\mathrm{B}, \mathrm{C}, \mathrm{AB}, \mathrm{AC}, \mathrm{BC}$, and $\mathrm{ABC}$ ) were studied plus the NONFBAP type constituting particles that do not fluoresce in any channel (e.g., channels A, B, and C). NON-FBAP concentrations are 1 order of magnitude higher than FBAP concentrations, and NON-FBAP hence traced WIBS total particles throughout all sampling events (Fig. 3a). Total FBAP concentrations also show similar behavior to the total particle concentration (Fig. 3a), which suggests that non-biological particles can be biasing the total FBAP concentration. The variability of the total FBAP concentration is mainly linked 
to type A and type B concentrations as overall they constitute the two largest fractions in the total FBAP concentration (Fig. 3b), and both FBAP types have previously misidentified non-biological particles as FBAP (Tropak and Schnaiter, 2013; Yu et al., 2016). As a result, our study considers the total FBAP concentration to be the upper limit and ABCtype concentration to be the lower limit of FBAP concentration in Metro Atlanta. Type B dominates the FBAP fractional composition (Fig. 3b), which has been linked to possible non-biological interferences from black carbon (Yu et al., 2016) and polycyclic aromatic hydrocarbons (PAHs) emitted from combustion sources. Total FBAP fraction ranges from $16 \%$ to $43 \%$, and $\mathrm{ABC}$ fraction ranges from $1.3 \%$ to $9.2 \%$ of the total particles in the 1 to $5 \mu \mathrm{m}$ size range. ABC-type fractions and $\mathrm{ABC}$-type concentrations are within the values observed by Tropak and Schnaiter (2013) using WIBS-4 in Karlsruhe, Germany, averaging $2.9 \times 10^{4} \mathrm{~m}^{-3}$ (when considering the sum of $\mathrm{AC}$ and $\mathrm{ABC}$ types) and constituting $7 \%$ of total coarse-mode particles $(0.8-16 \mu \mathrm{m})$.

ABC-type concentrations show an interesting variability throughout the 15 sampling events, as $\mathrm{ABC}$ reaches its maximum concentration on 14 April, on a warm and humid day after a rain event, concurrently when the FCM HNA population also reaches its highest concentration - strongly suggesting that $\mathrm{ABC}$ particles are fungal spores (Fig. 3a, Table 1). Furthermore, the WIBS high-resolution data in Fig. S24 show the enhancement of the $\mathrm{AB}$ and $\mathrm{ABC}$ types right after the beginning of the rain event on 13 April (18:00; night before sampling on 14 April) and are not correlated with NON-FBAP concentrations; FBAP concentration enhancement was previously linked to wet-ejected fungal spores (Huffman et al., 2013; Gosselin et al., 2016). Gosselin et al. (2016) used WIBS-3 in the Rocky Mountains, Colorado, showing ABC-type fractional composition enhances after rain events to dominate the total FBAP composition, and the enhancement is correlated with mannitol and arabitol concentrations (fungal spore tracers), which have been previously linked to Ascomycota and Basidiomycota spores emitted by the wet-ejection mechanism (Elbert et al., 2007). In addition, $\mathrm{ABC}$ types constitute a considerable fraction $(\sim 20 \%)$ of total FBAP during dry days in the Rocky Mountains, possibly because such highly vegetative environments maintain a high background of fungal spores (Huffman et al., 2013). However, urban environments like Metro Atlanta are not necessarily dominated by fungal spores, and its FBAP composition will be affected by the biological sources close to the city (e.g., forests), local emissions, and meteorology. The overall FBAP composition in Metro Atlanta (Fig. 3b) is dominated by type B (average fraction: $33 \pm 9 \%$ ), type A (average fraction: $22 \pm 5 \%$ ), and type $\mathrm{AB}$ (average fraction: $22 \pm 5 \%$ ) particles. Type ABC constitutes $12 \pm 6 \%$ of the total FBAP and reaches $30 \%$ on 14 April, comparable to values observed by Gosselin et al. (2016) in the Rocky Mountains. The dominance of type B particles has been observed in the polluted atmosphere of Nanjing, China, us- ing WIBS-4A where type B constituted $\sim 45 \%$ of the total PBAP and type $\mathrm{B}\left(\sim 2 \times 10^{6} \mathrm{~m}^{-3}\right)$ concentrations were up to 2 orders of magnitude higher than type A concentrations $\left(\sim 5 \times 10^{4} \mathrm{~m}^{-3}\right)$, suggesting a high likelihood of interference from abiotic particle sources. However, Metro Atlanta shows much lower total particle concentrations than Nanjing, China $\left(\sim 10^{7} \mathrm{~m}^{-3}\right)$, and type A and type B concentrations are within the same order of magnitude. Furthermore, Perring et al. (2015) have shown that type B particles constitute a considerable fraction of the total supermicron particles across the United States, being $\sim 15 \%$ and $\sim 25 \%$ over (altitude $>100 \mathrm{~m}$ ) the southeastern US and southwestern US, respectively. Total particle and NON-FBAP size distributions in Fig. 3c peaked at $\sim 1 \mu \mathrm{m}$. Similarly, type A, B, and AB size distributions (Fig. 3d) peaked close to $1 \mu \mathrm{m}$, showing that interferences by non-biological particles cannot be ruled out. However, ABC-type size distribution (light blue line, Fig. $3 \mathrm{~d}$ ) is dominated by $3-5 \mu \mathrm{m}$ particles, and ABC-type particles may have come from a different source to other FBAP types, as they get enhanced after rain events (e.g., 14 April; Table 1). Yu et al. (2016) also observed 4-6 $4 \mathrm{~m}$ ABC-type particles in the highly polluted Nanjing, China, but ABC-type bimodal size distributions showed a peak between 1 and $2 \mu \mathrm{m}$ and a second peak between 4 and $6 \mu \mathrm{m}$. In addition, ABC-type number fractions in Nanjing, China, correlated with black carbon mass fractions, suggesting a considerable influence by combustion-related particles, and no rain events occurred during the sampling period. The difference between Metro Atlanta and Nanjing, China, ABC-type size distributions suggests that $\mathrm{ABC}$ type is not influenced by combustion-related particles in Metro Atlanta. Overall, results show FBAP concentration $(1-5 \mu \mathrm{m})$ ranges from $10^{4}$ to $10^{5} \mathrm{~m}^{-3}$ in Metro Atlanta, and wet-ejected fungal spore concentration, detected by $\mathrm{ABC}$ type, can constitute up to $30 \%$ of the FBAP $(1-5 \mu \mathrm{m})$ after rain events.

\subsection{Correlation of HNA population with ABC type}

A quantitative comparison between WIBS-4A total particle and FCM total particle concentrations was subsequently performed and we focused the analysis to the 1 to $5 \mu \mathrm{m}$ size range as SpinCon sampling efficiency is reduced significantly above $5 \mu \mathrm{m}$ ( $\leq 14 \%$; Kesavan and Sagripanti, 2015). WIBS-4A and FCM total particle concentrations differed by about 1 order of magnitude (for optical diameter, $d_{0}$, greater than $1.5 \mu \mathrm{m}$ ) and particle concentration difference increased for particles with $d_{\mathrm{o}}<1.5 \mu \mathrm{m}$, as shown in the size distribution (geometrically averaged across the 15 SpinCon II sampling events) in Fig. 4a. The largest difference between WIBS-4A and uncorrected FCM size distributions seems to be related to SpinCon II having a cutoff size close to $1 \mu \mathrm{m}$, reducing significantly its sampling efficiency. Even with the observed difference in the magnitude of the concentrations between the two techniques, ABC-type and HNA concentrations were traced throughout all the sampling events, are 
(a)

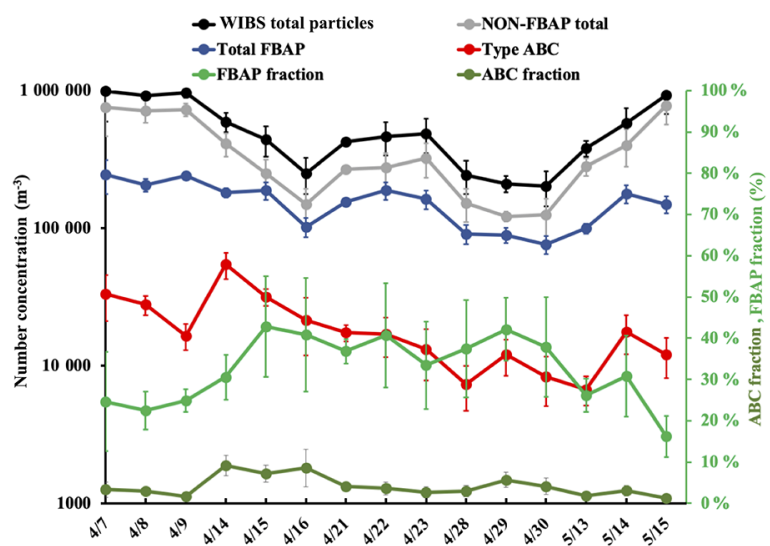

(c)

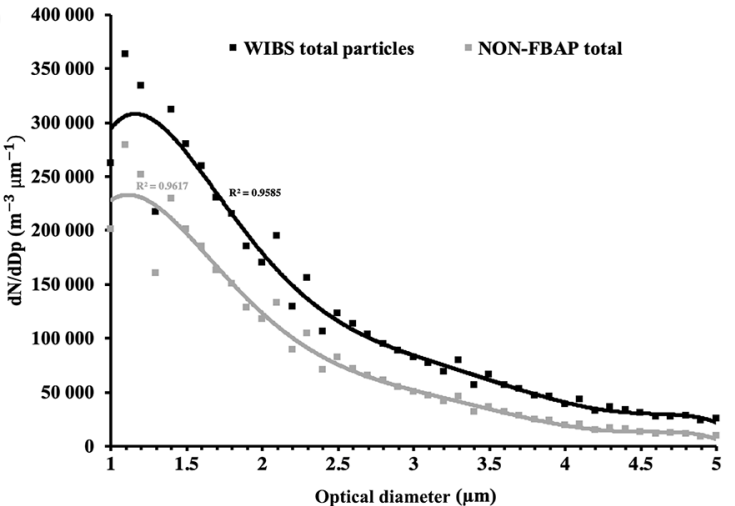

(b)

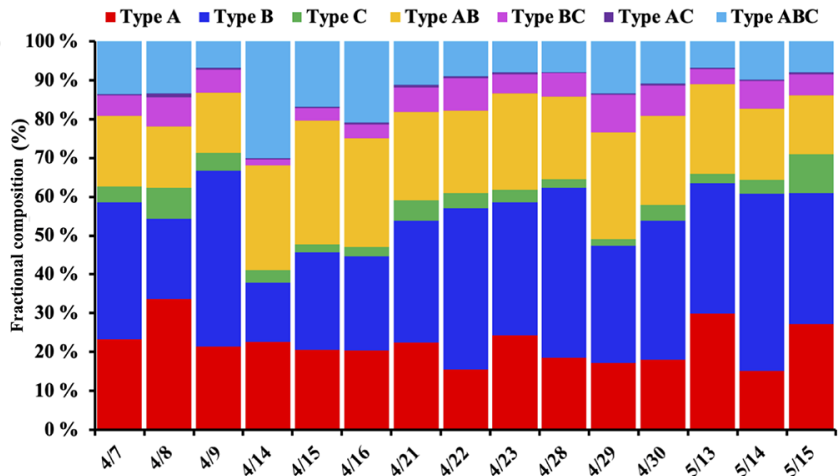

(d)

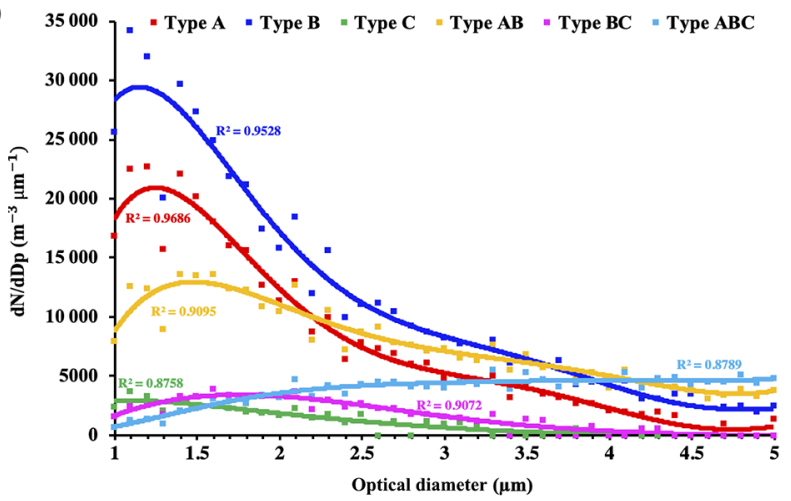

Figure 3. WIBS-4A $4 \mathrm{~h}$ (SpinCon II sampling time) averaged results of WIBS total particle, NON-FBAP, total FBAP, and type ABC concentrations on the left $y$ axis and ABC and FBAP fractions on the right $y$ axis for each SpinCon II sampling event in (a); $4 \mathrm{~h}$ averaged FBAP-type number concentration fractional composition for each SpinCon II sampling event in (b); 1 to $5 \mu \mathrm{m}$ WIBS total particles and NON-FBAP size distributions in (c) and 1 to $5 \mu \mathrm{m}$ size distributions for all FBAP types, except the AC type in (d). The AC type showed low statistics and constituted less than $1 \%$ of the total FBAP (not shown). Size distributions in (c) and (d) have been averaged over the 15 SpinCon II sampling events and constitute the overall size distributions during rooftop sampling events. Solid lines in (c) and (d) show sixth-degree polynomial regressions performed to FBAP the size distributions, including their respective correlation coefficients $\left(R^{2}\right)$.

moderately correlated $\left(R^{2}=0.40, P\right.$ value $=0.016 ;$ Fig. $\left.4 \mathrm{~b}\right)$, and showed similar size distributions in the 1 to $5 \mu \mathrm{m}$ range as shown in Fig. S12a. HNA and ABC type were both dominated by $3-5 \mu \mathrm{m}$ particles, and its seems both are detecting the same type of biological particle. In addition, $\mathrm{AB}$ type showed a weak correlation with HNA concentrations $\left(R^{2}=0.17\right)$, but their size distributions differed as type $\mathrm{AB}$ peaks close to $\sim 1 \mu \mathrm{m}$ (Fig. 3d). ABC is the only FBAP type showing a considerable correlation with the HNA population, and the LNA-AT population is not correlated with any FBAP type. Overall, $\mathrm{ABC}$ type and HNA correlation are important steps forward to better understand the effectiveness of WIBS4A FBAP categories to provide speciated PBAP concentrations in urban areas. ABC-type particles have shown substantial concentrations $\left(10^{4}-10^{5} \mathrm{~m}^{-3}\right.$; Perring et al., 2015; Ziemba et al., 2016) across the US. The highest ABC fraction of the total FBAP was observed in Panhandle, Florida, during an airborne study among multiple environments studied using WIBS-4A to sample from the California coast to central Florida, suggesting that $\mathrm{ABC}$-type particles are ubiqui- tous in the US (Perring et al., 2015). Previous studies (Healy et al., 2014; Huffman et al., 2013) have shown correlations between LIF technology (e.g., WIBS-4 and UV-APS) fluorescence channels and fungal spores number concentrations, especially during fungal spores invigoration after rain events. Healy et al. (2014) used WIBS-4 in Killarney National Park, Ireland (e.g., high vegetative rural area) finding correlations between channel B (FL2; $\left.R^{2}=0.29\right)$ and channel C (FL3; $R^{2}=0.38$ ) concentrations and fungal spores concentrations (collected by Sporewatch impactor and quantified by microscopy). Gosselin et al. (2016) observed stronger correlations between fungal spores (inferred from mannitol and arabitol concentrations) and WIBS-4 concentrations in the Rocky Mountains, but our study in Atlanta, GA was carried out in a completely different environment (e.g., highly populated urban environment). Now for the first time FCM HNA populations have shown a correlation with WIBS-4A $\mathrm{ABC}$ type and suggest that the $\mathrm{ABC}$ type category detects wet actively ejected fungal spores in Metro Atlanta (e.g., urban area). In addition, recent WIBS-4A experiments using 
pure cultures have shown $\mathrm{ABC}$ type detects well several fungal spores (e.g., Aspergillus Versicolor and Botrytis spp.) and small pollen grains, but detection may vary across instruments (Hernandez et al., 2016).

FCM concentrations were corrected based on correction factors $(\mathrm{CFs})$ calculated upon the comparison of $\mathrm{ABC}$ and HNA size distributions ( 1 to $5 \mu \mathrm{m}$ ) for each sampling event given (1) ABC-type and HNA population similar size distribution and number concentration ( 1 to $5 \mu \mathrm{m}$ ) correlation, and (2) WIBS-4A provides us with representative concentrations of airborne particle concentrations in Metro Atlanta after sampling losses are corrected (Sect. 3.2). Concentration correction factors were determined for each sampling episode by taking the quotient of the ABC type to HNA concentrations over the $1-5 \mu \mathrm{m}$ size range. The resulting size-dependent correction factor (Fig. S12b) was then applied to the FCM size distributions, giving the "corrected FCM" bioaerosol data (between 1 and $5 \mu \mathrm{m}$ ). Figure $4 \mathrm{a}$ shows that the corrected FCM total particle average size distribution traces WIBS4A size distribution, allowing us to correct for SpinCon II low collection efficiency and to better constrain the magnitude of FCM concentrations. Our approach to calculate the estimated collection efficiency (ECE) considers all the processes that affect the concentration of PBAP, from collection to final quantification in the FCM. Figure S12b compares Kesavan and Sagripanti (2015) collection efficiencies determined for SpinCon I and the estimated collection efficiency calculated upon the $\mathrm{CF}$ calculation $(\mathrm{ECE}=1 / \mathrm{CF})$ and shows the ECE of the SpinCon II is lower that Kesavan and Sagripanti (2015) below $3 \mu \mathrm{m}$ and performs better for particles above $3 \mu \mathrm{m}$, but above $3 \mu \mathrm{m}$ Kesavan and Sagripanti (2015) collection efficiency is within the uncertainty of our calculations. Our lower ECE values (Fig. S12b) for particles below $3 \mu \mathrm{m}$ can be related to the SpinCon sampling time as Kesavan and Sagripanti (2015) experiments were conducted in a short period of time (e.g., 10-15 min), and ours took place for $4 \mathrm{~h}$. The main mechanisms leading to below $3 \mu \mathrm{m}$ particle losses could be their re-aerosolization over time being lost through the blower exhaust of the SpinCon II (Fig. 1). Also, coagulation of small particles over time can not be ruled out, but future work is needed to study it. Although SpinCon/FCM results correction based on the HNA and ABC-type size distribution comparison effectively constrains the efficiency of the SpinCon/FCM analysis in this study, corrections are limited to the 1 to $5 \mu \mathrm{m}$ size range and must acknowledge that the specific sampling may stress cells and affect their detection.

\subsection{PBAP populations after collection/detection corrections}

After correction through the application of the ABC correction factors, FCM total particle concentrations ( 1 to $5 \mu \mathrm{m}$ average: $5.5 \times 10^{5} \pm 5.1 \times 10^{5} \mathrm{~m}^{-3}$; Fig. 5a) are within the same order of magnitude as WIBS-4A concentrations ( 1 to
$5 \mu \mathrm{m}$ average: $5.4 \times 10^{5} \pm 2.9 \times 10^{5} \mathrm{~m}^{-3}$; Fig. $3 \mathrm{a}$ ), and continue to exhibit substantial variability. The HNA (e.g., fungal spores) population showed a substantial invigoration during three sampling events (7, 14, and 15 April; Fig. 5a and b). To better understand the role of meteorology in PBAP composition, $24 \mathrm{~h}$ averaged temperature and relative humidity were used to express the prevailing temperature and relative humidity $(\mathrm{RH})$ during each sampling event, considering the residence time of microorganisms (e.g., bacteria and fungal spores) before sampling. Sampling events were classified into four regimes based on the average diurnal ( $24 \mathrm{~h}$ average) relative humidity and ambient temperature, with $T=18^{\circ} \mathrm{C}$ $\left(65^{\circ} \mathrm{F}\right)$ to differentiate between warm and cold days and $\mathrm{RH}$ $=70 \%$ to differentiate between humid and dry days. During the 15 sampling days, temperature ranged from 10.4 to $31.2^{\circ} \mathrm{C}$, and $\mathrm{RH}$ varied from $19.0 \%$ to $97.0 \%$ in Atlanta, GA (see Table S4 in the Supplement). The temperature and RH threshold values were chosen based on the observations and understanding that a combination of temperature and RH within these threshold values can significantly impact bioaerosol composition. For instance, humid and warm conditions may lead to the invigoration of fungal spores by wet ejection from plants (Ingold, 1971), on contrary, PBAP will get stressed when exposed to warm and dry conditions. The sampling times, RH, ambient temperature and meteorological categories of each SpinCon II sample are presented in Table 1.

Humid and warm days (7, 14, and 15 April; light green shaded areas in Fig. 5a) were characterized by well-defined HNA and LNA-AT populations. These sampling episodes had the highest average HNA (fungal spore) concentration $\left(4.0 \times 10^{4} \pm 1.3 \times 10^{4} \mathrm{~m}^{-3}\right)$ among the four meteorological regimes and during these sampling events HNA constituted $\geq 77 \%$ of the total PBAP. Among the humid and warm days (Fig. 5a and b), average LNA-AT, HNA, and "unclassified" bioaerosol compositions were $6.1 \%, 84.0 \%$, and $9.9 \%$, respectively, of the total PBAP number. Also, the humid and warm days occurred after rain events, which can be linked directly to the strong fungal spore invigoration (Huffman et al., 2013). Before sampling, early morning precipitation occurred during 14 and 15 April, as well as during the night of 6 April. Precipitation did not occur during sampling in any of the humid and warm days. The FCM results (Fig. S15ac) that display the PBAP population between 7 and 9 April show a disappearance of the (HNA) fungal spore population during the transition from a "humid and warm" day (7 April) to a "dry and warm" day (9 April). Figure 5b shows how the HNA contribution to the total PBAP goes down on 8 April when RH decreases and is undetected on 9 April. Furthermore, Fig. 6a-c show FL1 vs. SSC-A plots for 14 to 16 April consecutive sampling periods, where a marked increase in the LNA-AT concentration from 15 to 16 April goes together with a striking decrease in the HNA concentration. HNA fraction went down from $92.0 \%$ to $34.1 \%$ of the total PBAP and LNA-AT concentration went up from $3.8 \times 10^{3}$ to 

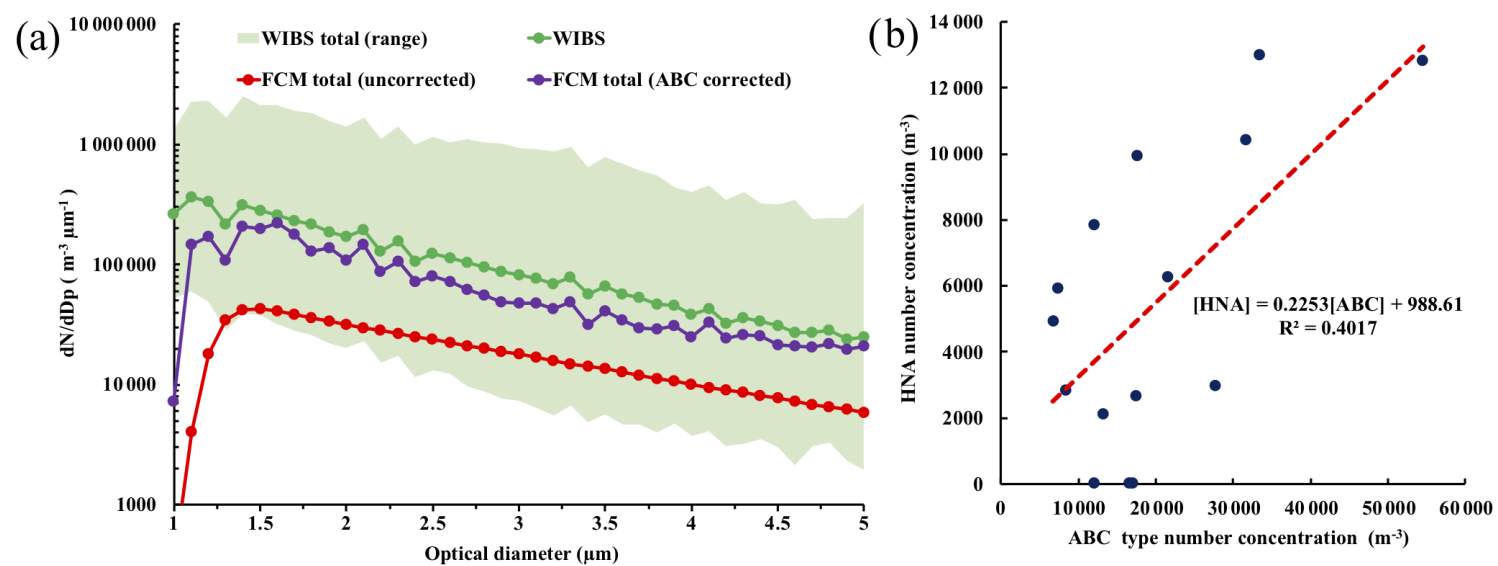

Figure 4. WIBS-4A, FCM-uncorrected, and FCM (ABC-corrected) total particle concentration (1 to $5 \mu \mathrm{m})$ average size distributions (geometrically averaged over the 15 SpinCon II sampling events), including the WIBS range ( \pm geometric standard deviation factor) in (a), and HNA and ABC-type concentration correlations in the 1 to $5 \mu \mathrm{m}$ range in (b), including its linear correlation in red.

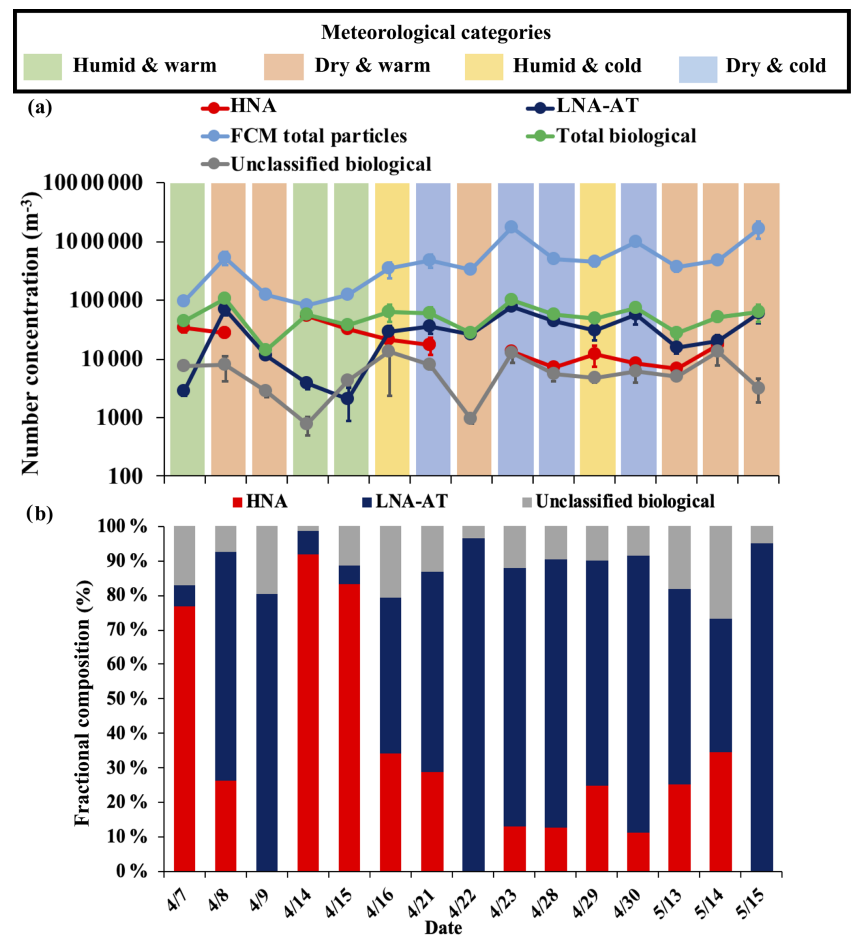

Figure 5. FCM total particle, HNA, LNA-AT, and total PBAP number concentrations in the 1 to $5 \mu \mathrm{m}$ range highlighting the prevailing meteorological category during each sampling event in (a); HNA and LNA-AT number concentration fractional compositions for each sampling event in (b).

$2.9 \times 10^{4} \mathrm{~m}^{-3}$. Humid and warm days had the lowest averaged PBAP concentration $\left(4.6 \times 10^{4} \pm 9.8 \times 10^{3} \mathrm{~m}^{-3}\right.$ in the 1 to $5 \mu \mathrm{m}$ range) among the four meteorological regimes, a possible effect of the bioaerosols being lost by wet scavenging, resulting in the enhancement of fungal spore contribution to the total PBAP number concentrations. The unclassified bi- ological particles concentration also showed its lowest contribution $\left(2.9 \times 10^{3} \mathrm{~m}^{-3} ; 9.9 \%\right)$ to the total PBAP number concentration during these events, when the HNA and LNA populations are best identified by the $2 \%$ contour plots.

Cold and humid days (16 and 29 April; light yellow shaded areas in Fig. 5a) also showed a well-defined HNA population, and HNA contributed on average $29.5 \pm 6.5 \%$ of the total PBAP concentration ( 1 to $5 \mu \mathrm{m}$ ). On 16 April drizzling took place by the end of the sampling period, but no accumulated rainfall was measured by the meteorological station. However, on 29 April, accumulated rainfall averaged 0.04 in. from 11:55 to 14:20. (Fig. S21). The similar HNA concentration between "humid and warm" and "humid and cold" days seen in Fig. 5a and the lower contribution of HNA to the total PBAP during the "humid and cold" days may be linked to previously suggested bacteria emissions by droplet soil impaction during rain events (Joung et al., 2017). Bacterial emission by soil impaction can increase airborne LNA-AT concentration and HNA (fungal spores) will have a lower contribution to the total PBAP even when the fungal spore concentration is high during rain events. Both cold and humid days showed a considerable difference in LNAAT contributions to the total PBAP concentration. On 16 and 29 April LNA-AT constituted $45.2 \%$ and $65.3 \%$ of the total PBAP concentration, respectively (Fig. 5b). The difference in the LNA-AT contribution to the total PBAP can be linked to the intensity of precipitation, as it shapes the composition (e.g., size and types) of microbes suspended in the atmosphere during the different stages of a rainfall (e.g., before, onset of, during, and after rainfall; Yue et al., 2016).

Six of the 15 sampling days were classified as warm and dry (8 April, 9 April, 22 April, 13 May, 14 May, 15 May; light orange shaded areas in Fig. 5a), and it did not rain before or during any of these days (Table 1). During warm and dry days, HNA had the lowest averaged concentration $\left(8.7 \times 10^{3} \pm 1.2 \times 10^{4} \mathrm{~m}^{-3}\right)$ among the four meteorological 

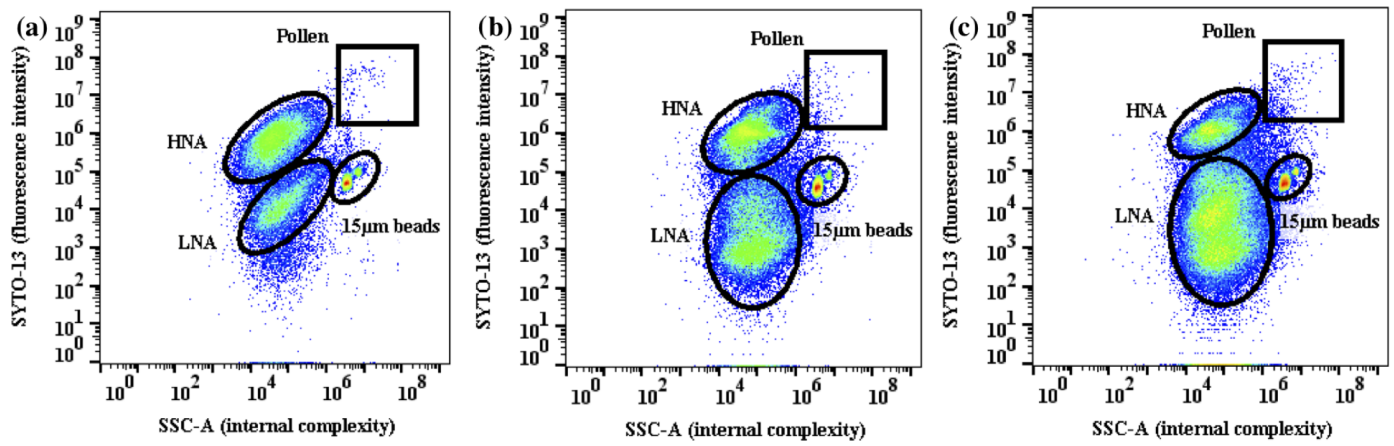

Figure 6. FL1-A vs. SSC-A FSC plots for (a) 14 April, (b) 15 April, and (c) 16 April. This period was characterized by a transition from humid and warm to humid and cold conditions (diurnal average $\mathrm{RH}=77 \%, T=22.5^{\circ} \mathrm{C}$ on 14 April; $\mathrm{RH}=84 \%, T=18.9^{\circ} \mathrm{C}$ on $15 \mathrm{April}$; and $\mathrm{RH}=86 \%, T=12.5^{\circ} \mathrm{C}$ on 16 April). The FCM plots during this transition period show a decrease in the fungal population and an increase in the LNA population. In each population, warmer colors represent higher particle concentrations.

categories. In addition, during 3 dry and warm days (9 April, 22 April, and 15 May), the HNA population was undetected. This behavior can be related to the fact that high RH drives fungal spore emissions by wet ejection, but soil wetness could also affect emissions because the HNA population was detected in other warm and dry days with comparable RH (Huffman et al., 2013; Gosselin et al., 2016). The air mass trajectories reaching Atlanta during each sampling event could also affect the biological particles composition. For example, on 22 April, when the HNA was undetected, the 500 and $100 \mathrm{~m} 72 \mathrm{~h}$ backward air mass trajectories reaching Atlanta came from the northwest (US-Canada border) at high altitudes and did not spend more than $24 \mathrm{~h}$ near the surface. This air mass could affect bioaerosol composition with minimal influence from local bioaerosol emissions. However, the enhancement or the depletion of the HNA population have not been linked to specific air masses trajectories. Overall, warm and dry days prevail during springtime in Atlanta, and LNA-AT contribution (average: $3.4 \times 10^{4} \pm 2.5 \times 10^{4} \mathrm{~m}^{-3}$ ) may represent the bioaerosol background of Atlanta.

Four of the 15 sampling days $(21,23,28$, and 30 April; light blue shaded areas in Fig. 5a) were characterized by cold and dry conditions (Table 1). PBAP were dominated by LNA-AT during these events, as can be seen in Fig. 7ac, where LNA populations are the dominant contributors to PBAP number. HNA population was diminished in Fig. 7a (21 April) and Fig. 7c (23 April) during cold and dry days and disappeared in Fig. 7b during a warm and dry day. Overall, HNA was detected during cold and dry days but showed lower contributions to the total PBAP number concentration than humid days. Among cold and dry days, the PBAP population ( 1 to $5 \mu \mathrm{m}$ ) was composed on average of $72.6 \pm 10.1 \%$ LNA-AT and $16.5 \pm 8.2 \%$ HNA. Cold and dry days had on average the highest LNA-AT $\left(5.3 \times 10^{4} \pm 1.8 \times 10^{4} \mathrm{~m}^{-3}\right)$ and total PBAP $\left(7.3 \times 10^{4} \pm 2.0 \times 10^{4} \mathrm{~m}^{-3}\right)$ number concentrations ( 1 to $5 \mu \mathrm{m})$ among the four meteorological categories, reaching the PBAP maximum concentration on 23 April (Fig. 5a).

\subsection{PBAP day-to-day variability in Metro Atlanta: FCM vs. WIBS}

Although WIBS and FCM possess different methodologies, they show similar trends, providing a good understanding of the daily variability of PBAP in Metro Atlanta. FCM PBAP fraction $(1$ to $5 \mu \mathrm{m})$ ranges from $3.8 \%$ to $69.2 \%$ of the total particles and the highest PBAP fraction $(69.2 \%)$ and HNA concentration is observed on 14 April $\left(5.25 \times 10^{4} \pm 5.89 \times\right.$ $\left.10^{3} \mathrm{~m}^{-3}\right)$. The total FBAP fraction (1 to $\left.5 \mu \mathrm{m}\right)$ ranges from $16 \%$ to $43 \%$, but it reaches its maximum on 15 April. However, $\mathrm{ABC}$ fraction of the total WIBS particle concentration ranges from $1.3 \%$ to $9.2 \%$ and it reaches its maximum on 14 April. Even when the magnitudes of the PBAP and FBAP fractions differ on average by a factor of $\sim 2$ throughout the sampling period, both techniques agree that an enhancement in the total biological particles takes place between 14 and 16 April. Given the uncertainty of the two methodologies, it is remarkable that there is such agreement between WIBS and FCM results.

Among the four meteorological categories, humid and warm days characterize the showing of the highest HNA, A-type, AB-type, and ABC-type concentrations, suggesting that $\mathrm{A}$ and $\mathrm{AB}$ types may also be related to wet-ejected fungal spores in Metro Atlanta. The observations possibly explain why the $\mathrm{ABC}$ fraction of the total FBAP on 7 April is not as high as on 14 and 15 April (Fig. 3b) and differs from the behavior observed by the HNA population on 7 April. The LNA-AT population does not show a correlation with any specific FBAP type and shows it highest concentrations during dry and cold days. In addition, LNA-AT concentrations are anticorrelated with type B concentrations (Fig. S19, correlation coefficient, $r=-0.59 ; R^{2}=0.30$ ) during dry (both cold and warm) days, when LNA-AT dominates the total PBAP concentration. Given that type B particles have 

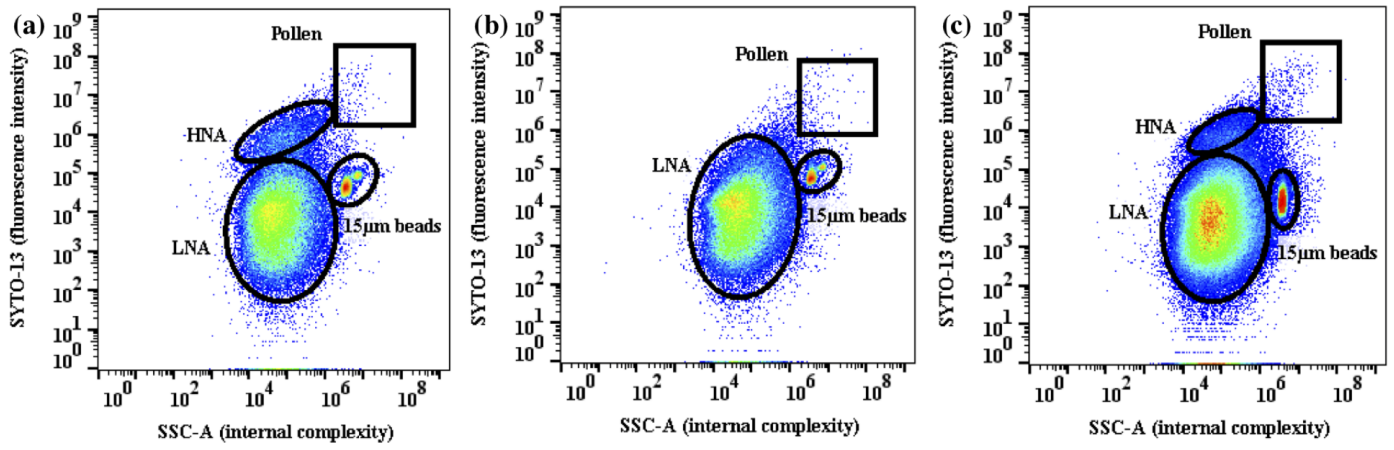

Figure 7. Similar to Fig. 6 but for (a) 21 April, (b) 22 April, and (c) 23 April, which was characterized by dryness and variability in temperature (diurnal average $\mathrm{RH}=43 \%, T=16.6^{\circ} \mathrm{C}$ on $21 \mathrm{April} ; \mathrm{RH}=41 \%, T=19.0^{\circ} \mathrm{C}$ on $22 \mathrm{April}$; and $\mathrm{RH}=48 \%, T=16.8^{\circ} \mathrm{C}$ on 23 April). Note the disappearance of the fungal spore population on the warmest day (22 April).

been previously correlated with abiotic particles (e.g., black carbon) in urban environments (Yue et al., 2017), LNA-AT and type B anticorrelation suggests that LNA-AT particles may in fact represent a heterogeneous bioaerosol population. That LNA-AT is not correlated with any FBAP type gives rise to two possibilities: (1) if the LNA-AT population is mainly composed of bacteria or agglomerated bacteria, then it is possible that they are detected by multiple FBAP types and are not attributed specifically to one of them; (2) the intrinsic fluorescence of LNA-AT particles is too low and a high fraction of them is abiotic. It is challenging to determine which PBAP types each WIBS FBAP type is mainly detecting. Based on WIBS-4A results in Metro Atlanta, ABC type detects wet-ejected fungal spores, but it is still unclear which PBAP types are detected by the other FBAP types or whether they just capture a high fraction of non-biological particles. FBAP types and WIBS total particle correlations in Fig. S17 show that all FBAP types are correlated with WIBS total particles, but $\mathrm{ABC}$ and $\mathrm{AB}$ types show the lowest correlations (type $\mathrm{AB}: R^{2}=0.101$; type $\mathrm{ABC}: R^{2}=0.1266$ ).

Figure 8 shows FCM total PBAP (black line), ABC type (light green), FL1 (channel A; dark green line), and total FBAP (blue line) concentrations, where the FL1 concentration ([FL1]) constitutes the sum of the number concentrations of types $\mathrm{A}, \mathrm{AB}, \mathrm{AC}$, and $\mathrm{ABC}$ $([\mathrm{FL} 1]=[\mathrm{A}]+[\mathrm{AB}]+[\mathrm{AC}]+[\mathrm{ABC}]$; Gabey et al., 2011; Healy et al., 2014). Throughout the April-May 2015 sampling events, total PBAP concentrations (1 to $5 \mu \mathrm{m})$ were mainly constrained between the FL1 and ABC-type concentrations, suggesting that FL1 and $\mathrm{ABC}$ type represent the upper- and lower-bound PBAP concentrations in Metro Atlanta, respectively. It also important to highlight that FCM PBAP concentrations are closer to the ABC-type concentrations before 16 April when the HNA population dominates, but then after 16 April FCM PBAP concentrations are closer to FL1 concentrations when LNA-AT starts to dominate the total PBAP concentration. In addition, Fig. 8 shows that total

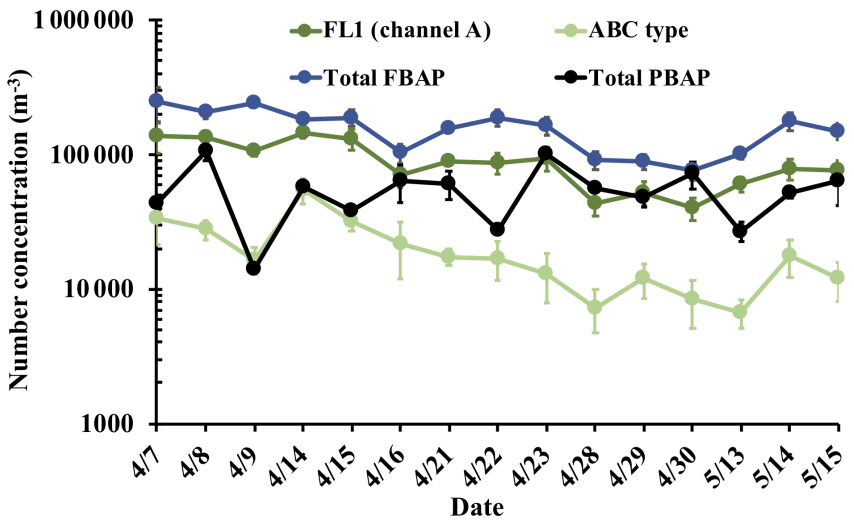

Figure 8. WIBS-4A total FBAP, FL1 and ABC type, and FCM total particle number concentrations in the 1 to $5 \mu \mathrm{m}$ range for each sampling event from 7 April to 15 May 2015.

FBAP (sum of types A, B, C, AB, AC, and ABC) exceeds the (corrected) PBAP concentrations in Metro Atlanta.

\section{Conclusions}

In this study we presented the development and testing of an effective FCM protocol to identify and quantify bioaerosol populations. The FCM protocol, designed to constrain any particle accumulation due to cleaning or by fluid supplies, successfully quantified the day-to-day variability of bioaerosols in the Metro Atlanta area. It is the first FCM study to detect well-defined LNA (low nucleic acid) and HNA (high nucleic acid) atmospheric biological populations under different meteorological scenarios. FCM results show dynamic bioaerosol populations in Atlanta leading to a $84.0 \%$ of HNA (wet-ejected fungal spores) and $6.1 \%$ LNAAT contribution to the PBAP number ( 1 to $5 \mu \mathrm{m}$ range), respectively, during humid and warm days after rain events. However, LNA-AT dominates warm and cold dry days, constituting $72 \%$ of the PBAP number concentration. 
WIBS-4A and SpinCon II collocated sampling showed that the HNA and ABC-type concentrations are well correlated $\left(R^{2}=0.40\right)$ and display similar size distributions. We therefore conclude that both instruments detect the same particles, and used empirical collection/detection efficiency factors to correct the FCM size distributions and concentrations in the 1 to $5 \mu \mathrm{m}$ diameter range. WIBS-4A and FCM results suggest that Metro Atlanta PBAP concentrations range between $10^{4}$ and $10^{5} \mathrm{~m}^{-3}$ ( 1 to $5 \mu \mathrm{m}$ ), and they can constitute a substantial fraction of coarse-mode particle concentration (WIBS-4A: 43\%; FCM: 69\%), comparable to the PBAP coarse-mode fraction in highly vegetated environments. The FCM LNA-AT population, possibly containing bacterial cells, did not correlate with any FBAP type. The fact that the LNA-AT population is not correlated with a specific FBAP type suggests that it may be particularly challenging to use LIF techniques to distinguish bioaerosols with low intrinsic autofluorescence from non-biological particles, especially given the heterogeneities introduced by the large biodiversity of airborne microbes. The possible influence of abiotic particles in the LNA-AT population can also explain the lack of correlation between LNA-AT and FBAP types given that the FCM threshold approach does not ensure total exclusion of abiotic particles. In addition, the unspecific binding of SYTO-13 to abiotic particles cannot be ruled out in the LNA-AT population. FCM comparison between atmospheric and pure culture samples showed lower SYTO-13 fluorescence intensities in the atmospheric samples and suggests a degradation in the genetic material of PBAP, possibly caused by the limited nutrients and strong stress prevailing in the atmosphere, which further challenge the ability of LIF to distinguish LNA-AT.

In summary, this study has shown for the first time that FCM can effectively identify, quantify, and study the daily variability of heterogeneous PBAP populations (e.g., HNA, LNA-AT, and pollen) with different genetic material content in atmospheric samples. We also show that a number of FCM and WIBS-4A populations are largely correlated and therefore can be used to identify the nature of the FBAP detected in the latter. Our results finally show that the detection and quantification of bacterial cells in atmospheric samples remains a challenging task and is best achieved through the combination of techniques.

Data availability. Data used to generate the figures can be found in the Supplement.

Supplement. The supplement related to this article is available online at: https://doi.org/10.5194/acp-20-1817-2020-supplement.

Author contributions. ArN, AtN, KTK, and MB conceived the study. ArN, NDR, and SMW developed the modified biosampler.
ArN and NDR developed the FCM analysis and sampling protocol. ArN and NDR carried out measurements, and SW helped with analysis of the biological samples. LDZ and BA provided the WIBS and helped with its setup and initial data analysis procedure. ArN and AtN worked on the analysis, wrote codes to interpret the data, and developed the analysis protocol to combine the FCM and WIBS analysis outlined here. ArN and AtN wrote the paper, and all the authors contributed with comments and modified text.

Competing interests. The authors declare that they have no conflict of interest.

Acknowledgements. We acknowledge support from a Georgia Power Faculty Scholar chair, a Cullen-Peck Faculty Fellowship, a Dreyfus Foundation Postdoctoral Fellowship in Environmental Chemistry, NASA, and a NASA Earth System Science Fellowship (grant no. 80NSSC17K0434). We also acknowledge support from project PyroTRACH (ERC-2016-COG) funded from H2020EU.1.1. - Excellent Science - European Research Council (ERC), project ID 726165. We also thank Rodney Weber for helpful suggestions on the SpinCon II flow calibration.

Financial support. This research has been supported by the Camille and Henry Dreyfus Foundation (Postdoctoral Fellowship in Environmental Chemistry), the National Aeronautics and Space Administration (Earth System Science Fellowship (grant no. 80NSSC17K0434)) and the European Research Council, H2020 Research Infrastructures (PyroTRACH (grant no. 726165)).

Review statement. This paper was edited by Anne Perring and reviewed by three anonymous referees.

\section{References}

Amato, P., Joly, M., Schaupp, C., Attard, E., Möhler, O., Morris, C. E., Brunet, Y., and Delort, A.-M.: Survival and ice nucleation activity of bacteria as aerosols in a cloud simulation chamber, Atmos. Chem. Phys., 15, 6455-6465, https://doi.org/10.5194/acp15-6455-2015, 2015.

Augustin, S., Wex, H., Niedermeier, D., Pummer, B., Grothe, H., Hartmann, S., Tomsche, L., Clauss, T., Voigtländer, J., Ignatius, K., and Stratmann, F.: Immersion freezing of birch pollen washing water, Atmos. Chem. Phys., 13, 10989-11003, https://doi.org/10.5194/acp-13-10989-2013, 2013.

Bacsi, A., Choudhury, B. K., Dharajiya, N., Sur, S., and Boldogh, I.: Subpollen particles: carriers of allergenic proteins and oxidases, J. Allergy Clin. Immun., 118, 844-850, https://doi.org/10.1016/j.jaci.2006.07.006, 2006.

Baillie, L. and Read, T. D.: Bacillus anthracis, a bug with attitude!, Curr. Opin. Microbiol., 4, 78-81, https://doi.org/10.1016/S13695274(00)00168-5, 2001.

Bauer, H., Claeys, M., Vermeylen, R., Schueller, E., Weinke, G., Berger, A., and Puxbaum, H.: Arabitol and mannitol as tracers 
for the quantification of airborne fungal spores, Atmos. Environ., 42, 588-593, https://doi.org/10.1016/j.atmosenv.2007.10.013, 2008a.

Bauer, H., Schueller, E., Weinke, G., Berger, A., Hitzenberger, R., Marr, I. L., and Puxbaum, H.: Significant contributions of fungal spores to the organic carbon and to the aerosol mass balance of the urban atmospheric aerosol, Atmos. Environ., 42, 5542-5549, https://doi.org/10.1016/j.atmosenv.2008.03.019, 2008b.

Bochdansky, A. B., Clouse, M. A., and Herndl, G. J.: Eukaryotic microbes, principally fungi and labyrinthulomycetes, dominate biomass on bathypelagic marine snow, ISME J., 11, 362, https://doi.org/10.1038/ismej.2016.113, 2016.

Bouvier, T., Del Giorgio, P. A., and Gasol, J. M.: A comparative study of the cytometric characteristics of high and low nucleic-acid bacterioplankton cells from different aquatic ecosystems, Environ. Microbiol., 9, 2050-2066, https://doi.org/10.1111/j.1462-2920.2007.01321.x, 2007.

Burrows, S. M., Elbert, W., Lawrence, M. G., and Pöschl, U.: Bacteria in the global atmosphere - Part 1: Review and synthesis of literature data for different ecosystems, Atmos. Chem. Phys., 9, 9263-9280, https://doi.org/10.5194/acp-9-9263-2009, 2009.

Chen, P. S. and Li, C. S.: Bioaerosol characterization by flow cytometry with fluorochrome, J. Environ. Monitor., 7, 950-959, https://doi.org/10.1039/b505224f, 2005.

Chi, M.-C. and Li, C.-S.: Fluorochrome in Monitoring Atmospheric Bioaerosols and Correlations with Meteorological Factors and Air Pollutants, Aerosol Sci. Tech., 41, 672-678, https://doi.org/10.1080/02786820701383181, 2007.

Comas-Riu, J. and Vives-Rego, J.: Cytometric monitoring of growth, sporogenesis and spore cell sorting in Paenibacillus polymyxa (formerly Bacillus polymyxa), J. Appl. Microbiol., 92, 475-481, https://doi.org/10.1046/j.1365-2672.2002.01549.x, 2002.

Crawford, I., Ruske, S., Topping, D. O., and Gallagher, M. W.: Evaluation of hierarchical agglomerative cluster analysis methods for discrimination of primary biological aerosol, Atmos. Meas. Tech., 8, 4979-4991, https://doi.org/10.5194/amt-8-4979-2015, 2015.

Darrow, L. A., Hess, J., Rogers, C. A., Tolbert, P. E., Klein, M., and Sarnat, S. E.: Ambient pollen concentrations and emergency department visits for asthma and wheeze, J. Allergy Clin. Immun., 130, 630-638, https://doi.org/10.1016/j.jaci.2012.06.020, 2012.

DeLeon-Rodriguez, N.: Microbes in the atmosphere: prevalence, species composition, and relevance to cloud formation School of Biology Georgia Institute of Technology, 129 pp., available at: http://hdl.handle.net/1853/55517 (last access: 9 August 2018), 2015.

DeLeon-Rodriguez, N., Lathem, T. L., Rodriguez-R, L. M., Barazesh, J. M., Anderson, B. E., Beyersdorf, A, J., Ziemba, L. D., Bergin, M., Nenes, A., and Konstantinidis, K. T.: Microbiome of the upper troposphere: Species composition and prevalence, effects of tropical storms, and atmospheric implications, P. Natl. Acad. Sci. USA, 110, 2575-2580, 2013.

Delort, A.-M. and Amato, P.: Microbiology of aerosols, 167-168, John Wiley \& Sons Inc., Hoboken, NJ, USA, https://doi.org/10.1002/9781119132318, 2018

Després, V. R., Alex Huffman, J., Burrows, S. M., Hoose, C., Safatov, A. S., Buryak, G., Fröhlich-Nowoisky, J., Elbert, W., Andreae, M. O., Pöschl, U., and Jaenicke, R.: Primary biological aerosol particles in the atmosphere: a review, Tellus B, 64, 1, https://doi.org/10.3402/tellusb.v64i0.15598, 2012.

Díaz, M., Herrero, M., García, L. A., and Quirós, C.: Application of flow cytometry to industrial microbial bioprocesses, Biochem. Eng. J., 48, 385-407, https://doi.org/10.1016/j.bej.2009.07.013, 2010.

Eckenrode, H. M., Jen, S.-H., Han, J., Yeh, A.-G., and Dai, H.-L.: Adsorption of a Cationic Dye Molecule on Polystyrene Microspheres in Colloids:? Effect of Surface Charge and Composition Probed by Second Harmonic Generation, J. Phys. Chem. B, 109, 4646-4653, https://doi.org/10.1021/jp045610q, 2005.

Elbert, W., Taylor, P. E., Andreae, M. O., and Pöschl, U.: Contribution of fungi to primary biogenic aerosols in the atmosphere: wet and dry discharged spores, carbohydrates, and inorganic ions, Atmos. Chem. Phys., 7, 4569-4588, https://doi.org/10.5194/acp-74569-2007, 2007.

Fröhlich-Nowoisky, J., Kampf, C. J., Weber, B., Huffman, J. A., Pöhlker, C., Andreae, M. O., Lang-Yona, N., Burrows, S. M., Gunthe, S. S., Elbert, W., Su, H., Hoor, P., Thines, E., Hoffmann, T., Després, V. R., and Pöschl, U.: Bioaerosols in the Earth system: Climate, health, and ecosystem interactions, Atmos. Res., 182, 346-376, https://doi.org/10.1016/j.atmosres.2016.07.018, 2016.

Gabey, A. M., Gallagher, M. W., Whitehead, J., Dorsey, J. R., Kaye, P. H., and Stanley, W. R.: Measurements and comparison of primary biological aerosol above and below a tropical forest canopy using a dual channel fluorescence spectrometer, Atmos. Chem. Phys., 10, 4453-4466, https://doi.org/10.5194/acp10-4453-2010, 2010.

Gabey, A. M., Stanley, W. R., Gallagher, M. W., and Kaye, P. H.: The fluorescence properties of aerosol larger than $0.8 \mu \mathrm{m}$ in urban and tropical rainforest locations, Atmos. Chem. Phys., 11, 5491-5504, https://doi.org/10.5194/acp-11-5491-2011, 2011.

Gosselin, M. I., Rathnayake, C. M., Crawford, I., Pöhlker, C., Fröhlich-Nowoisky, J., Schmer, B., Després, V. R., Engling, G., Gallagher, M., Stone, E., Pöschl, U., and Huffman, J. A.: Fluorescent bioaerosol particle, molecular tracer, and fungal spore concentrations during dry and rainy periods in a semi-arid forest, Atmos. Chem. Phys., 16, 15165-15184, https://doi.org/10.5194/acp-16-15165-2016, 2016.

Goudie, A. S.: Desert dust and human health disorders, Environ. Int., 63, 101-113, https://doi.org/10.1016/j.envint.2013.10.011, 2014.

Griffin, D. W., Kellogg, C. A., Garrison, V. H., Lisle, J. T., Borden, T. C., and Shinn, E. A.: Atmospheric microbiology in the northern Caribbean during African dust events, Aerobiologia, 19, 143-157, https://doi.org/10.1023/B:AERO.0000006530.32845.8d, 2003.

Grote, M., Valenta, R., and Reichelt, R.: Abortive pollen germination: A mechanism of allergen release in birch, alder, and hazel revealed by immunogold electron microscopy, J. Allergy Clin. Immun., 111, 1017-1023, https://doi.org/10.1067/mai.2003.1452, 2003.

Guarín, F. A., Abril, M. A. Q., Alvarez, A., and Fonnegra, R.: Atmospheric pollen and spore content in the urban area of the city of Medellin, Colombia, Hoehnea, 42, 9-19, 2015.

Guindulain, T., Comas, J., and Vives-Rego, J.: Use of nucleic acid dyes SYTO-13, TOTO-1, and YOYO-1 in the study of Es- 
cherichia coli and marine prokaryotic populations by flow cytometry, Appl. Environ. Microb., 63, 4608-4611, 1997.

Harrison, R. M., Jones, A. M., Biggins, P. D. E., Pomeroy, N., Cox, C. S., Kidd, S. P., Hobman, J. L., Brown, N. L., and Beswick, A.: Climate factors influencing bacterial count in background air samples, Int. J. Biometeorol., 49, 167-178, https://doi.org/10.1007/s00484-004-0225-3, 2005.

Healy, D. A., O'Connor, D. J., and Sodeau, J. R.: Measurement of the particle counting efficiency of the "Waveband Integrated Bioaerosol Sensor" model number 4 (WIBS-4), J. Aerosol Sci., 47, 94-99, https://doi.org/10.1016/j.jaerosci.2012.01.003, 2012.

Healy, D. A., Huffman, J. A., O’Connor, D. J., Pöhlker, C., Pöschl, U., and Sodeau, J. R.: Ambient measurements of biological aerosol particles near Killarney, Ireland: a comparison between real-time fluorescence and microscopy techniques, Atmos. Chem. Phys., 14, 8055-8069, https://doi.org/10.5194/acp14-8055-2014, 2014.

Hernandez, M., Perring, A. E., McCabe, K., Kok, G., Granger, G., and Baumgardner, D.: Chamber catalogues of optical and fluorescent signatures distinguish bioaerosol classes, Atmos. Meas. Tech., 9, 3283-3292, https://doi.org/10.5194/amt-9-3283-2016, 2016.

Hill, S. C., Mayo, M. W., and Chang, R. K.: Fluorescence of bacteria, pollens, and naturally occurring airborne particles: excitation/emission spectra, Army Research Lab Adelphi Md Computational And Information Sciences Directorate, U.S. Army Research Laboratory, Adelphi, MD, USA, available at: https://apps. dtic.mil/dtic/tr/fulltext/u2/a494347.pdf (last access: 25 November 2019), 2009.

Hoose, C. and Möhler, O.: Heterogeneous ice nucleation on atmospheric aerosols: a review of results from laboratory experiments, Atmos. Chem. Phys., 12, 9817-9854, https://doi.org/10.5194/acp-12-9817-2012, 2012.

Hoose, C., Kristjánsson, J. E., and Burrows, S. M.: How important is biological ice nucleation in clouds on a global scale?, Environ. Res. Lett., 5, 024009, https://doi.org/10.1088/17489326/5/2/024009, 2010.

Huffman, J. A., Treutlein, B., and Pöschl, U.: Fluorescent biological aerosol particle concentrations and size distributions measured with an Ultraviolet Aerodynamic Particle Sizer (UVAPS) in Central Europe, Atmos. Chem. Phys., 10, 3215-3233, https://doi.org/10.5194/acp-10-3215-2010, 2010.

Huffman, J. A., Prenni, A. J., DeMott, P. J., Pöhlker, C., Mason, R. H., Robinson, N. H., Fröhlich-Nowoisky, J., Tobo, Y., Després, V. R., Garcia, E., Gochis, D. J., Harris, E., MüllerGermann, I., Ruzene, C., Schmer, B., Sinha, B., Day, D. A., Andreae, M. O., Jimenez, J. L., Gallagher, M., Kreidenweis, S. M., Bertram, A. K., and Pöschl, U.: High concentrations of biological aerosol particles and ice nuclei during and after rain, Atmos. Chem. Phys., 13, 6151-6164, https://doi.org/10.5194/acp13-6151-2013, 2013.

Ingold, C. T.: Fungal spores. Their liberation and dispersal, Oxford, Clarendon Press, 302 pp., 1971.

Joung, Y., Ge, Z., and Buie, C.: Bioaerosol generation by raindrops on soil, Nat. Commun., 8, 14668, https://doi.org/10.1038/ncomms14668, 2017.

Kesavan, J. and Sagripanti, J. L.: Evaluation criteria for bioaerosol samplers, Environ. Sci. Process Impacts, 17, 638-645, https://doi.org/10.1039/c4em00510d, 2015.
Lange, J. L., Thorne, P. S., and Lynch, N.: Application of flow cytometry and fluorescent in situ hybridization for assessment of exposures to airborne bacteria, Appl. Environ. Microb., 63, 1557-1563, 1997.

Lebaron, P., Servais, P., Agogue, H., Courties, C., and Joux, F.: Does the high nucleic acid content of individual bacterial cells allow us to discriminate between active cells and inactive cells in aquatic systems?, Appl. Environ. Microb., 67, 1775-1782, https://doi.org/10.1128/AEM.67.4.1775-1782.2001, 2001.

Li, D.-W. and Kendrick, B.: A year-round study on functional relationships of airborne fungi with meteorological factors, Int. J. Biometeorol., 39, 74-80, 1995.

Liang, L., Engling, G., Cheng, Y., Duan, F., Du, Z., and He, K.: Rapid detection and quantification of fungal spores in the urban atmosphere by flow cytometry, J. Aerosol Sci., 66, 179-186, https://doi.org/10.1016/j.jaerosci.2013.08.013, 2013.

Lin, H., Gomez, I., and Meredith, J. C.: Pollenkit Wetting Mechanism Enables Species-Specific Tunable Pollen Adhesion, Langmuir, 29, 3012-3023, https://doi.org/10.1021/la305144z, 2013.

Longo, A. F., Ingall, E. D., Diaz, J. M., Oakes, M., King, L. E., Nenes, A., Mihalopoulos, N., Violaki, K., Avila, A., Benitez-Nelson, C. R., Brandes, J., McNulty, I., and Vine, D. J.: P-NEXFS analysis of aerosol phosphorus delivered to the Mediterranean Sea, Geophys. Res. Lett., 41, 4043-4049, https://doi.org/10.1002/2014GL060555, 2014.

Mage, P. L., Csordas, A. T., Brown, T., Klinger, D., Eisenstein, M., Mitragotri, S., Hawker, C., and Soh, H. T.: Shape-based separation of synthetic microparticles, Nat. Mater., 18, 82-89, https://doi.org/10.1038/s41563-018-0244-9, 2019.

Mathaes, R., Winter, G., Engert, J., and Besheer, A.: Application of different analytical methods for the characterization of nonspherical micro-and nanoparticles, Int. J. Pharm., 453, 620-629, 2013.

Monier, J. M. and Lindow, S. E.: Pseudomonas syringae Responds to the Environment on Leaves by Cell Size Reduction, Phytopathology, 93, 1209-1216, https://doi.org/10.1094/PHYTO.2003.93.10.1209, 2003.

Morris, C. E., Conen, F., Alex Huffman, J., Phillips, V., Pöschl, U., and Sands, D. C.: Bioprecipitation: a feedback cycle linking earth history, ecosystem dynamics and land use through biological ice nucleators in the atmosphere, Glob. Change Biol., 20, 341-351, https://doi.org/10.1111/gcb.12447, 2014.

Müller, S. and Nebe-von-Caron, G.: Functional single-cell analyses: flow cytometry and cell sorting of microbial populations and communities, FEMS Microbiol. Rev., 34, 554-587, https://doi.org/10.1111/j.1574-6976.2010.00214.x, 2010.

Myriokefalitakis, S., Nenes, A., Baker, A. R., Mihalopoulos, N., and Kanakidou, M.: Bioavailable atmospheric phosphorous supply to the global ocean: a 3-D global modeling study, Biogeosciences, 13, 6519-6543, https://doi.org/10.5194/bg-13-6519-2016, 2016.

Nir, R., Yisraeli, Y., Lamed, R., and Sahar, E.: Flow cytometry sorting of viable bacteria and yeasts according to beta-galactosidase activity, Appl. Environ. Microbiol., 56, 3861-3866, 1990.

Oliveira, M., Ribeiro, H., Delgado, J. L., and Abreu, I.: The effects of meteorological factors on airborne fungal spore concentration in two areas differing in urbanisation level, Int. J. Biometeorol., 53, 61-73, https://doi.org/10.1007/s00484-008-0191-2, 2009.

Ortiz-Martínez, M. G., Rodríguez-Cotto, R. I., Ortiz-Rivera, M. A., Pluguez-Turull, C. W., and Jiménez-Vélez, B. D.: Linking en- 
dotoxins, African dust $\mathrm{PM}_{10}$ and asthma in an urban and rural environment of Puerto Rico, Mediat. Inflamm., 2015, 784212, https://doi.org/10.1155/2015/784212, 2015.

Pan, Y.-L., Santarpia, J. L., Ratnesar-Shumate, S., Corson, E., Eshbaugh, J., Hill, S. C., Williamson, C. C., Coleman, M., Bare, C., and Kinahan, S.: Effects of ozone and relative humidity on fluorescence spectra of octapeptide bioaerosol particles, J. Quant. Spectrosc. Ra., 133, 538-550, https://doi.org/10.1016/j.jqsrt.2013.09.017, 2014.

Perring, A. E., Schwarz, J., Baumgardner, D., Hernandez, M., Spracklen, D., Heald, C., Gao, R., Kok, G., McMeeking, G., and McQuaid, J.: Airborne observations of regional variation in fluorescent aerosol across the United States, J. Geophys. Res.-Atmos., 120, 1153-1170, https://doi.org/10.1002/2014JD022495, 2015.

Pöhlker, C., Huffman, J. A., and Pöschl, U.: Autofluorescence of atmospheric bioaerosols - fluorescent biomolecules and potential interferences, Atmos. Meas. Tech., 5, 37-71, https://doi.org/10.5194/amt-5-37-2012, 2012.

Pöhlker, C., Huffman, J. A., Förster, J.-D., and Pöschl, U.: Autofluorescence of atmospheric bioaerosols: spectral fingerprints and taxonomic trends of pollen, Atmos. Meas. Tech., 6, 3369-3392, https://doi.org/10.5194/amt-6-3369-2013, 2013.

Pöschl, U.: Atmospheric Aerosols: Composition, Transformation, Climate and Health Effects, Angew. Chem. Int. Edit., 44, 75207540, https://doi.org/10.1002/anie.200501122, 2005.

Robinson, N. H., Allan, J. D., Huffman, J. A., Kaye, P. H., Foot, V. E., and Gallagher, M.: Cluster analysis of WIBS single-particle bioaerosol data, Atmos. Meas. Tech., 6, 337-347, https://doi.org/10.5194/amt-6-337-2013, 2013.

Rödiger, S., Ruhland, M., Schmidt, C., Schröder, C., Grossmann, K., Böhm, A., Nitschke, J., Berger, I., Schimke, I., and Schierack, P.: Fluorescence Dye Adsorption Assay to Quantify Carboxyl Groups on the Surface of Poly(methyl methacrylate) Microbeads, Anal. Chem., 83, 3379-3385, https://doi.org/10.1021/ac103277s, 2011.

Saari, S., Reponen, T., and Keskinen, J.: Performance of Two Fluorescence-Based Real-Time Bioaerosol Detectors: BioScout vs. UVAPS, Aerosol Sci. Tech., 48, 371-378, https://doi.org/10.1080/02786826.2013.877579, 2014.

Šantl-Temkiv, T., Amato, P., Gosewinkel, U., Thyrhaug, R., Charton, A., Chicot, B., Finster, K., Bratbak, G., and Löndahl, J.: High-Flow-Rate Impinger for the Study of Concentration, Viability, Metabolic Activity, and Ice-Nucleation Activity of Airborne Bacteria, Environ. Sci. Technol., 51, 11224-11234, 10.1021/acs.est.7b01480, 2017.

Savage, N. J., Krentz, C. E., Könemann, T., Han, T. T., Mainelis, G., Pöhlker, C., and Huffman, J. A.: Systematic characterization and fluorescence threshold strategies for the wideband integrated bioaerosol sensor (WIBS) using size-resolved biological and interfering particles, Atmos. Meas. Tech., 10, 4279-4302, https://doi.org/10.5194/amt-10-4279-2017, 2017.

Shapiro, H. M.: Practical flow cytometry, John Wiley \& Sons, Hoboken, NJ, USA, 2005.

Standaert-Vitse, A., Aliouat-Denis, C.-M., Martinez, A., Khalife, S., Pottier, M., Gantois, N., Dei-Cas, E., and Aliouat, E. M.: SYTO-13, a Viability Marker as a New Tool to Monitor In Vitro Pharmacodynamic Parameters of Anti-Pneumocystis Drugs, PLoS One, 10, e0130358, https://doi.org/10.1371/journal.pone.0130358, 2015.

Sullivan, S. C., Hoose, C., Kiselev, A., Leisner, T., and Nenes, A.: Initiation of secondary ice production in clouds, Atmos. Chem. Phys., 18, 1593-1610, https://doi.org/10.5194/acp-181593-2018, 2018.

Taylor, P. E., Jacobson, K. W., House, J. M., and Glovsky, M. M.: Links between pollen, atopy and the asthma epidemic, Int. Arch. Allergy Imm., 144, 162-170, https://doi.org/10.1159/000103230, 2007.

Tropak, E. and Schnaiter, M.: Fluorescent biological aerosol particles measured with the Waveband Integrated Bioaerosol Sensor WIBS-4: laboratory tests combined with a one year field study, Atmos. Chem. Phys., 13, 225-243, https://doi.org/10.5194/acp13-225-2013, 2013.

Troussellier, M., Courties, C., Lebaron, P., and Servais, P.: Flow cytometric discrimination of bacterial populations in seawater based on SYTO 13 staining of nucleic acids, FEMS Microbiol. Ecol., 29, 319-330, https://doi.org/10.1016/S01686496(99)00026-4, 1999.

Tzur, A., Moore, J. K., Jorgensen, P., Shapiro, H. M., and Kirschner, M. W.: Optimizing Optical Flow Cytometry for Cell Volume-Based Sorting and Analysis, PLoS ONE, 6, e16053, https://doi.org/10.1371/journal.pone.0016053, 2011.

Van Dilla, M. A., Langlois, R. G., Pinkel, D., Yajko, D., and Hadley, W. K.: Bacterial characterization by flow cytometry, Science, 220, 620-622, 1983.

von der Weiden, S.-L., Drewnick, F., and Borrmann, S.: Particle Loss Calculator - a new software tool for the assessment of the performance of aerosol inlet systems, Atmos. Meas. Tech., 2, 479-494, https://doi.org/10.5194/amt-2-479-2009, 2009.

Wang, Y., Hammes, F., De Roy, K., Verstraete, W., and Boon, N.: Past, present and future applications of flow cytometry in aquatic microbiology, Trends Biotechnol., 28, 416-424, https://doi.org/10.1016/j.tibtech.2010.04.006, 2010.

Wu, Y.-H., Chan, C.-C., Rao, C. Y., Lee, C.-T., Hsu, H.-H., Chiu, Y.-H., and Chao, H. J.: Characteristics, determinants, and spatial variations of ambient fungal levels in the subtropical Taipei metropolis, Atmos. Environ., 41, 2500-2509, https://doi.org/10.1016/j.atmosenv.2006.11.035, 2007.

Yu, X., Wang, Z., Zhang, M., Kuhn, U., Xie, Z., Cheng, Y., Pöschl, U., and $\mathrm{Su}, \mathrm{H} .:$ Ambient measurement of fluorescent aerosol particles with a WIBS in the Yangtze River Delta of China: potential impacts of combustion-related aerosol particles, Atmos. Chem. Phys., 16, 11337-11348, https://doi.org/10.5194/acp-16-113372016, 2016.

Yue, S., Ren, H., Fan, S., Sun, Y., Wang, Z., and Fu, P.: Springtime precipitation effects on the abundance of fluorescent biological aerosol particles and HULIS in Beijing, Sci. Rep.-UK, 6, 29618, https://doi.org/10.1038/srep29618, 2016.

Yue, S., Ren, H., Fan, S., Wei, L., Zhao, J., Bao, M., Hou, S., Zhan, J., Zhao, W., Ren, L., Kang, M., Li, L., Zhang, Y., Sun, Y., Wang, Z., and Fu, P.: High Abundance of Fluorescent Biological Aerosol Particles in Winter in Beijing, China, ACS Earth and Space Chemistry, 1, 493-502, https://doi.org/10.1021/acsearthspacechem.7b00062, 2017.

Zhen, H., Han, T., Fennell, D. E., and Mainelis, G.: Release of free DNA by membrane-impaired bacterial aerosols due to 
aerosolization and air sampling, Appl. Environ. Microb., 79, 7780-7789, https://doi.org/10.1128/AEM.02859-13, 2013.

Ziemba, L. D., Beyersdorf, A. J., Chen, G., Corr, C. A., Crumeyrolle, S. N., Diskin, G., Hudgins, C., Martin, R., Mikoviny, T., Moore, R., Shook, M., Thornhill, K. L., Winstead, E. L., Wisthaler, A., and Anderson, B. E.: Airborne observations of bioaerosol over the Southeast United States using a Wideband Integrated Bioaerosol Sensor, J. Geophys. Res.-Atmos., 121, 85068524, https://doi.org/10.1002/2015JD024669, 2016. 